\title{
Nonlinear Control of a Multi-Link Aerial System and ASEKF-based Disturbances Compensation
}

\author{
Jose J. Castillo Zamora ${ }^{1, *}$, Juan Escareno ${ }^{2}$, Islam Boussaada ${ }^{1,3}$, Joanny Stephant ${ }^{2}$, Ouiddad Labanni-Igbida ${ }^{2}$
}

\begin{abstract}
The actual paper presents the modeling and control of a multi-link unmanned aerial system whose dynamics is computed by means of the Euler-Lagrange energy-based approach. The aforementioned system is subjected to lumped disturbances which comprise external disturbances and parametric uncertainties. An Augmented-State Extended Kalman Filter intended to estimate endogenous and exogenous uncertainties is conceived and trajectory-tracking controller fulfilling Lyapunov asymptotic stability is synthesized. A simulation stage is conducted to validate the effectiveness of the proposal.
\end{abstract}

Index Terms-Multi-Link Unmanned Aerial System, Augmented-State Extended Kalman Filter, Disturbances Estimation/Compensation, Nonlinear Control, Multi-cargo Aerial Transportation.

\section{INTRODUCTION}

A MID the current technological surge related to interactive UAS have enlarged the application spectrum. It includes high-precision weather monitoring, swarm-based remote sensing, search and rescue, reconnaissance, parcel delivering, homeland security and surveillance, precision agriculture, disaster assessment, and infrastructure inspection [1], [2], [3]. The wide variety of prior works on aerial transportation witnesses the evolution on this field ranging from single [4], [5], [6] to multi-drone configurations [7], [8]. The latter reveals plenty of scientific and technological challenges with enormous potential regarding the industrial sector.

The research reported in [4] addresses aerial cargo transportation as a disturbed navigation case and thus a robust controller is used to fulfill the trajectory-tracking objective. Similarly, [5] exposes a robust trajectory-tracking strategy applied to a quadrotor. In the former, the cargo is acquired by a fully actuated one degree-of-freedom (DOF) robotic arm while in the latter it is considered as a free-swinging load.

Regarding flight performance of cable- and rod-suspended loads aerial transport, the knowledge or estimation of parasitic dynamics exerted on the rotorcraft remains a priority, as in [6] where a Learning Automata methodology is implemented for estimation purposes. In order to cope with multiple cargo a logical solution is to use multiple vehicles [7], [8].

Limited transport capacity of a single vehicle can be overcome through an alternative inter-linked configuration composed by several aerial sub-systems. While multi-linked aerial systems

\footnotetext{
${ }^{1}$ J. Castillo and I. Boussaada are with LS2A IPSA, Ivry-sur-Seine, France and L2S, Université Paris Sud-CNRS-CentraleSupelec, Université Paris Saclay, Gif-sur-Yvette, France.

2 J. Escareno, J. Stephant and O. Lagbanni are with ENSIL-ENSCI, Limoges, CNRS, XLIM, UMR 7252, 87280 Limoges France.

${ }^{3}$ I. Boussaada is with INRIA Saclay, Equipe DISCO

* Corresponding author: jose.castillo@ipsa.fr
}

concept has a great promise, it also comes with complex issues in terms of mechanics, dynamic couplings, synchronization, and collective interaction [1], [2], [3], [9], [8]. An interesting feature of this concept is the enhanced shape adaptability similar to that of snake-like amphibious robots [10], [11], and to that of the serial manipulator robots [12], [13].

Parallel robots have been recently integrated to aerial robots ([14], [15]). Inspired on the advantages of these kind of manipulators, the authors of [16] propose an aerial system having three quadrotors acting as the actuators. It is also presented, the dynamic model of the robot whose validation is carried out at numerical level.

To the best of our knowledge few works have been reported in literature regarding multi-link aerial systems for cargo transportation. In [17] a dual-rotor multi-link aerial system exhibiting an in-flight morphing capacity via the pose control is introduced. Prior work of these authors includes a multirotor aerial vehicle with two-dimensional multi-links to perform maneuvers withing cluttered spaces [18]. Likewise, in [19], is addressed the path planning strategy in continuity to [17], [18] An alternative perspective is adopted by [20] which addresses the transportation problem considering multiple cargo morphing aerial robot to successfully cope with the center of gravity $(\mathrm{CoG})$ shifting resulting from the loads/vehicles motion.

Taking inspiration from serial configurations as manipulators robots or trains, the authors of [21] and [22] detail the modeling and control of an aerial serial kinematic chain of $n$ quadrotors liked via $n-1$ rigid rods. Respectively, it is presented a robust control strategy to solve the problem of multi-cargo acquisition and transportation, and the implementation of a linear Kalman filter (LKF) considering an augmented state to enhance the robustness of the transportation task control while tracking a time-based trajectory. In this case, the augmented state stands for the inter-vehicle dynamic couplings and disturbances coming from loads motion.

Multi-sensor fusion based on extended Kalman Filter (EKF) has been used for pose estimation of aerial systems to ensure a smooth and reliable aerial transformation [23]. In some works, efforts are focused to estimate the surrounding environment influence. Wind effects are estimated by observers, stochastic or deterministic as ([24], while [25] presents an estimation strategy to compensate the external wrench exerted on the aerial multi-link robot while estimating external forces.

Different state-estimation techniques as sliding-mode based observers [26] are also used to improve the performance of the system, however, due to its performance the Kalman Filter is widely used [27], [28], [29], [30], [31], [32], [33], [34], [35]. In this paper, we consider a multi-link aerial system that 
is intended to track a time-based trajectory while rejecting parametric and external disturbances during a multiple-load transportation task. The main contributions of the actual work are listed below:

- The presentation of an alternative concept of aerial multilink rotorcraft meant to transport multiple cargo.

- The control synthesis considering the longitudinal dynamics is detailed and applied to the proposed flying robot in presence of structured and unstructured uncertainties.

- Since the aerial system is nonlinear, an augmented-state extended Kalman-Filter (AS-EKF) is implemented. The model's covariance matrix structure is deduced based on the uncertainties characterization/profile with respect to the full nonlinear model.

- A detailed numerical study is conducted to evaluate the effectiveness of the proposed overall control-estimation strategy. A realistic scenario is considered including sensors operational specifications in terms of noise and faults.

The paper is outlined as follows: Section II presents the specifics of the proposed aerial system. The dynamic model is explained throughout the Section III. Section IV is devoted to the mathematical model extension which defines the augmented state-space representation to introduce Section $\mathrm{V}$ entails the model's uncertainties analysis that shapes the AS-EKF estimation algorithm. The Lyapunov-based control strategy is proposed in Section VI and it is validated via numerical simulation in Section VII. The set of results are discussed in Section VIII, while concluding remarks alongside forthcoming research are presented in Section IX. Finally, within the Appendix section, pertinent system properties and the linearization regarding the observability analysis are presented.

\section{System Description}

The system proposed herein consists in three quadrotors physically interlinked through two rods (Fig.1). Aerial transportation, manipulation and deployment is possible through 1-DoF robotic manipulators, whose joints are located at the CoG of the rods [16], [21], [22].

For pedagogical purposes, we restrict our analysis to the longitudinal dynamics (see Fig.2) where $d_{i}$ with $i=1,2,3$ stands for the aerial subsystems, $l_{j} \in \mathbb{R}^{+}$corresponds to the

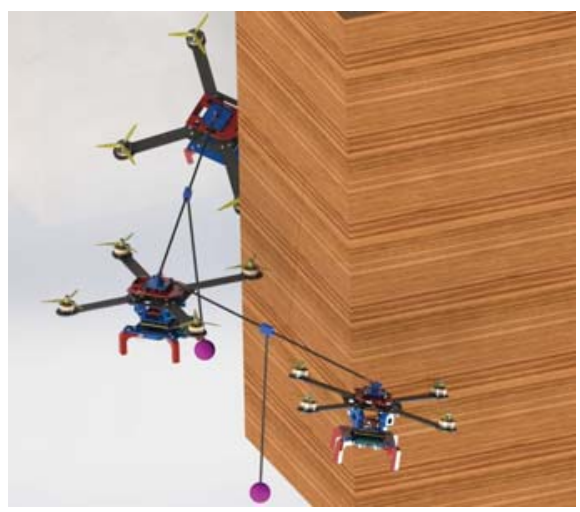

Figure 1: 3D CAD Scheme of the multi-link system

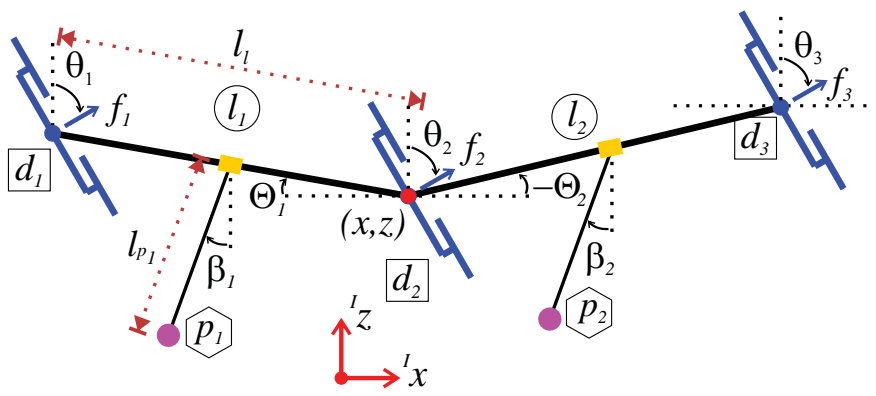

Figure 2: Longitudinal simplified scheme of the multi-link system

$j^{\text {th }}$-link and $p_{j}$ indicates the pendulum-like robotic-arm system whose length is denoted as $l_{p_{j}}$ with $j=1,2$.

The attitude of the $i^{t h}$-vehicle is denoted by $\theta_{i} \in \mathbb{R}$, while $\Theta_{j} \in \mathbb{R}$ represents the $j^{t h}$-linkage's attitude between the $i^{\text {th }}$ and $(i-1)^{t h}$ rotorcrafts w.r.t. the $\mathrm{x}$-axis of the inertial frame. The rods interlinking the rotorcrafts are assumed rigid with mass $m_{l} \in \mathbb{R}^{+}$and length $l_{l} \in \mathbb{R}^{+}$, thus its moment of inertia corresponds to $I_{l}=m_{l} l_{l}^{2} / 12$. The $j^{\text {th }}$ massless rod pendulum carries a mass $m_{p_{j}} \in \mathbb{R}^{+}$whose angular displacement is $\beta_{j} \in \mathbb{R}$. The mass of a single rotorcraft is denoted as $m_{r} \in \mathbb{R}^{+}$ and moment of inertia $I_{r} \in \mathbb{R}^{+}$. The force $f_{i} \in \mathbb{R}$, drives the $i^{t h}$ vehicle within the longitudinal space. In fact, the rotorcrafts serve as the actuators thus they are considered in the Lagrangian-based modeling of the system [21], [22].

The reference tracking point of the system w.r.t. the inertial frame corresponds to the $\mathrm{CoG}$ of the $d_{2}$ rotorcraft whose position is denoted as $\boldsymbol{\xi}_{r_{2}}=\left[\begin{array}{ll}x & z\end{array}\right]^{T} \in \mathbb{R}^{2}$, moreover, from Fig.2, the positions of the UAVs, $\boldsymbol{\xi}_{r_{i}}=\left[\begin{array}{ll}x_{r_{i}} & z_{r_{i}}\end{array}\right]^{T} \in \mathbb{R}^{2}$ and those of the rigid links, $\boldsymbol{\xi}_{l_{j}}=\left[\begin{array}{ll}x_{l_{j}} & z_{l_{j}}\end{array}\right]^{T} \in \mathbb{R}^{2}$, and the payloads, $\boldsymbol{\xi}_{p_{j}}=\left[\begin{array}{ll}x_{p_{j}} & z_{p_{j}}\end{array}\right]^{T} \in \mathbb{R}^{2}$, are defined as:

$$
\begin{array}{rlrl}
\boldsymbol{\xi}_{r_{1}} & =\left[\begin{array}{l}
x-l_{l} C_{\Theta_{1}} \\
z+l_{l} S_{\Theta_{1}}
\end{array}\right], & \boldsymbol{\xi}_{r_{3}}=\left[\begin{array}{l}
x+l_{l} C_{\Theta_{2}} \\
z-l_{l} S_{\Theta_{2}}
\end{array}\right], \\
\boldsymbol{\xi}_{l_{1}}=\left[\begin{array}{l}
x-0.5 l_{l} C_{\Theta_{1}} \\
z+0.5 l_{l} S_{\Theta_{1}}
\end{array}\right], & \boldsymbol{\xi}_{l_{2}}=\left[\begin{array}{c}
x+0.5 l_{l} C_{\Theta_{2}} \\
z-0.5 l_{l} S_{\Theta_{2}}
\end{array}\right], \\
\boldsymbol{\xi}_{p_{1}}=\boldsymbol{\xi}_{l_{1}}-\left[\begin{array}{l}
l_{p_{1}} S_{\beta_{1}} \\
l_{p_{1}} C_{\beta_{1}}
\end{array}\right], & \boldsymbol{\xi}_{p_{2}}=\boldsymbol{\xi}_{l_{2}}-\left[\begin{array}{l}
l_{p_{2}} S_{\beta_{2}} \\
l_{p_{2}} C_{\beta_{2}}
\end{array}\right]
\end{array}
$$

where $S_{(\star)}=\sin (\star)$ and $C_{(\star)}=\cos (\star)$.

By differentiating Eqs .(1), (2) and (3) w.r.t. time and assuming the parameters of the system to be time-invariant, it is straightforward to compute the velocity of each component.

\section{DYNAMICAL MODEL}

To compute the dynamics of the system by the means of the Euler-Lagrange formalism, the total kinetic, $\mathscr{K} \in \mathbb{R}^{+}$, and potential, $\mathscr{U} \in \mathbb{R}$, energies define the Lagrangian $\mathscr{L}=$ $\mathscr{K}-\mathscr{U} \in \mathbb{R}$ in such a manner that the term $\mathscr{K}$ comprises the translational and rotational energies of the quadrotors, the 
links and the payloads, and the term $\mathscr{U}$ gathers the potential energy of each element, as follows

$$
\begin{aligned}
& \mathscr{K}=\sum_{i=1}^{3}\left(\frac{m_{r}}{2} \dot{\boldsymbol{\xi}}_{r_{i}}^{2}+\frac{I_{r}}{2} \dot{\boldsymbol{\theta}}_{i}^{2}\right)+\sum_{j=1}^{2}\left(\frac{m_{l}}{2} \dot{\boldsymbol{\xi}}_{l_{j}}^{2}+\frac{I_{l}}{2} \dot{\Theta}_{j}^{2}+\frac{m_{p_{j}} \dot{\boldsymbol{\xi}}_{p_{j}}^{2}}{2}\right) \\
& \mathscr{U}=g\left[m_{r} \sum_{i=1}^{3} z_{r_{i}}+\sum_{j=1}^{2}\left(m_{l} z_{l_{j}}+m_{p_{j}} z_{p_{j}}\right)\right]
\end{aligned}
$$

where $\dot{\boldsymbol{\xi}}_{(\star)}^{2}=\dot{\boldsymbol{\xi}}_{(\star)}^{T} \dot{\boldsymbol{\xi}}_{(\star)}$ and $g \in \mathbb{R}^{+}$is the gravity acceleration. The Euler-Lagrange equation follows the form

$$
\frac{d}{d t} \frac{\partial}{\partial \dot{q}_{i}} \mathscr{L}-\frac{\partial}{\partial q_{i}} \mathscr{L}=\tau_{q_{i}}
$$

implies the definition of a vector $\mathbf{q} \in \mathbb{R}^{6}$ of generalized coordinates $q_{i} \in \mathbb{R}$ (with $i=1,2, \ldots, 6$ ) that for this specific case of study: $q_{1}=x, q_{2}=z, q_{3}=\Theta_{1}, q_{4}=\Theta_{2}, q_{5}=\beta_{1}$ and $q_{6}=\beta_{2}$. The term $\tau_{q_{i}} \in \mathbb{R}$ stands for the external forces/torques. The equations of motion of the system are comprised in the expression of the form:

$$
M(\mathbf{q}) \ddot{\mathbf{q}}+C(\mathbf{q}, \dot{\mathbf{q}}) \dot{\mathbf{q}}+\mathbf{G}(\mathbf{q})=\tau
$$

where the inertial matrix $M(\mathbf{q}) \in \mathbb{R}^{6 \times 6}$ and the vector of gravitational terms $\mathbf{G}(\mathbf{q}) \in \mathbb{R}^{6}$ are defined as:

$$
\begin{aligned}
M(\mathbf{q}) & =\left[\begin{array}{cccccc}
m_{11} & 0 & m_{13} & m_{14} & m_{15} & m_{16} \\
0 & m_{22} & m_{23} & m_{24} & m_{25} & m_{26} \\
m_{31} & m_{32} & m_{33} & 0 & m_{35} & 0 \\
m_{41} & m_{42} & 0 & m_{44} & 0 & m_{46} \\
m_{51} & m_{52} & m_{53} & 0 & m_{55} & 0 \\
m_{61} & m_{62} & 0 & m_{64} & 0 & m_{66}
\end{array}\right] \\
\mathbf{G}(\mathbf{q}) & =g\left[\begin{array}{llllll}
0 & m_{22} & m_{23} & m_{24} & m_{25} & m_{26}
\end{array}\right]^{T}
\end{aligned}
$$

and the Coriolis and centripetal effects matrix $C(\mathbf{q}, \dot{\mathbf{q}}) \in \mathbb{R}^{6 \times 6}$ is computed such that it satisfies $\dot{M}(\mathbf{q})=C(\mathbf{q}, \dot{\mathbf{q}})+C(\mathbf{q}, \dot{\mathbf{q}})^{T}$. Due to the symmetry property of $M(\mathbf{q})$, it is sufficient to define the elements below, where $\vartheta_{j}=\Theta_{j}-\beta_{j}$.

$$
\begin{array}{ll}
m_{11}=3 m_{r}+2 m_{l}+m_{p_{1}}+m_{p_{2}} & m_{22}=m_{11} \\
m_{13}=l_{l}\left[m_{r}+0.5\left(m_{l}+m_{p_{1}}\right)\right] S_{\Theta_{1}} & m_{15}=-m_{p_{1}} l_{p_{1}} C_{\beta_{1}} \\
m_{14}=-l_{l}\left[m_{r}+0.5\left(m_{l}+m_{p_{2}}\right)\right] S_{\Theta_{2}} & m_{16}=-m_{p_{2}} l_{p_{2}} C_{\beta_{2}} \\
m_{23}=l_{l}\left[m_{r}+0.5\left(m_{l}+m_{p_{1}}\right)\right] C_{\Theta_{1}} & m_{25}=m_{p_{1}} l_{p_{1}} S_{\beta_{1}} \\
m_{24}=-l_{l}\left[m_{r}+0.5\left(m_{l}+m_{p_{2}}\right)\right] C_{\Theta_{2}} & m_{26}=m_{p_{2}} l_{p_{2}} S_{\beta_{2}} \\
m_{33}=l_{l}^{2}\left[m_{r}+0.25\left(m_{l}+m_{p_{1}}\right)\right]+I_{l} & m_{35}=-0.5 m_{p_{1}} l_{l} l_{p_{1}} S_{\vartheta_{1}} \\
m_{44}=l_{l}^{2}\left[m_{r}+0.25\left(m_{l}+m_{p_{2}}\right)\right]+I_{l} & m_{46}=0.5 m_{p_{2}} l_{l} l_{p_{2}} S_{\vartheta_{2}} \\
m_{55}=m_{p_{1}} l_{p_{1}}^{2} & m_{66}=m_{p_{2}} l_{p_{2}}^{2}
\end{array}
$$

The vector $\tau=\left[\begin{array}{llllll}\tau_{x} & \tau_{z} & \tau_{\Theta_{1}} & \tau_{\Theta_{2}} & \tau_{\beta_{1}} & \tau_{\beta_{2}}\end{array}\right]^{T} \in \mathbb{R}^{6}$ in Eq.(5) is composed by the control inputs produced by the quadrotor and manipulator arm actuators $\mathbf{u}_{\tau} \in \mathbb{R}^{6}$ and the disturbances $\boldsymbol{\rho}=\left[\begin{array}{llllll}\rho_{x} & \rho_{z} & \rho_{\Theta_{1}} & \rho_{\Theta_{2}} & \rho_{\beta_{1}} & \rho_{\beta_{2}}\end{array}\right]^{T} \in \mathbb{R}^{6}$ caused by external unmodeled phenomena, in this sense $\tau=\mathbf{u}_{\tau}+\boldsymbol{\rho}$ where the vector of control inputs $\mathbf{u}_{\tau}$ depends on the geometry of the system, the forces $f_{1}, f_{2}, f_{3}$ exerted by the vehicles, the orientation of the aircrafts $\theta_{1}, \theta_{2}, \theta_{3}$ and the torques $\tau_{s_{1}}, \tau_{s_{2}} \in \mathbb{R}$ produced by the servomotors to manipulate the robotic arms, moreover, we assume that the dynamics of the actuators (aircrafts and servomotors) is significantly faster than that of the overall system, thus it follows that:

$$
\mathbf{u}_{\tau}=\left[\begin{array}{c}
f_{1} S_{\theta_{1}}+f_{2} S_{\theta_{2}}+f_{3} S_{\theta_{3}} \\
f_{1} C_{\theta_{1}}+f_{2} C_{\theta_{2}}+f_{3} C_{\theta_{3}} \\
\frac{l_{l}}{2}\left(f_{1} C_{\Theta_{1}-\theta_{1}}-f_{2} C_{\Theta_{1}-\theta_{2}}\right) \\
\frac{l_{l}}{2}\left(f_{2} C_{\Theta_{2}-\theta_{2}}-f_{3} C_{\Theta_{2}-\theta_{3}}\right) \\
\tau_{s_{1}} \\
\tau_{s_{2}}
\end{array}\right]
$$

By following Eq.(4) and taking into account $\theta_{i}$ instead of $q_{i}$, the rotational motion equation of the rotorcrafts is defined as $I_{r} \ddot{\theta}_{i}=\tau_{r_{i}}$ as we consider a frictionless motion between the inter-connected elements. $\tau_{r_{i}} \in \mathbb{R}$ is the control torque of the $i^{\text {th }}$ vehicle.

\section{Model Extension: Augmented State REPRESENTATION}

Based on Eq. (5), the dynamics of the multi-link system is expressed as

$$
\ddot{\mathbf{q}}=M(\mathbf{q})^{-1}\left[\mathbf{u}_{\tau}+\boldsymbol{\rho}-C(\mathbf{q}, \dot{\mathbf{q}}) \dot{\mathbf{q}}-\mathbf{G}(\mathbf{q})\right]
$$

The latest yields to a state space representation of the system as follows:

$$
\frac{d}{d t} \boldsymbol{\chi}=\left[\begin{array}{c}
\mathbf{0}^{\star} \\
M(\mathbf{q})^{-1}\left[\mathbf{u}_{\tau}+\boldsymbol{\rho}-C(\mathbf{q}, \dot{\mathbf{q}}) \dot{\mathbf{q}}-\mathbf{G}(\mathbf{q})\right]
\end{array}\right]
$$

where $\mathbf{0}^{\star} \in \mathbb{R}^{6}$ is the zero vector and $\boldsymbol{\chi}=\left[\mathbf{q}^{T} \dot{\mathbf{q}}^{T}\right]^{T} \in \mathbb{R}^{12}$ that can be extended to include the external disturbances such that Eq. (10) becomes:

$$
\frac{d}{d t} \boldsymbol{\chi}^{e}=\left[\begin{array}{c}
\boldsymbol{0}^{\star} \\
\boldsymbol{\vartheta}(\mathbf{q})^{-1}\left[\mathbf{u}_{\tau}+\boldsymbol{\rho}-C(\mathbf{q}, \dot{\mathbf{q}}) \dot{\mathbf{q}}-\mathbf{G}(\mathbf{q})\right]
\end{array}\right]
$$

with $\boldsymbol{\chi}^{e}=\left[\begin{array}{lll}\mathbf{q}^{T} & \dot{\mathbf{q}}^{T} \boldsymbol{\rho}^{T}\end{array}\right]^{T} \in \mathbb{R}^{18}$. Notice that $\mathbf{q}, \dot{\mathbf{q}}$ and $\boldsymbol{\rho}$ are functions of time and that the dynamics of the disturbances is modeled as a random walk process with zero mean (Gaussian) $\boldsymbol{\vartheta}(t)=\left[\begin{array}{llllll}\vartheta_{x} & \vartheta_{z} & \vartheta_{\Theta_{1}} & \vartheta_{\Theta_{2}} & \vartheta_{\beta_{1}} & \vartheta_{\beta_{2}}\end{array}\right]^{T} \in \mathbb{R}^{6}$. For instance, such system can be rewritten in the form:

$$
\dot{\boldsymbol{\chi}}^{e}=\mathbf{F}\left(\boldsymbol{\chi}^{e}, \mathbf{U}\right)+J \boldsymbol{\vartheta}
$$

with

$$
\begin{aligned}
& \mathbf{F}\left(\boldsymbol{\chi}^{e}, \mathbf{U}\right)=\left[\begin{array}{c}
\mathbf{0}^{\star} \\
M(\mathbf{q})^{-1}[\underset{\mathbf{U}}{\boldsymbol{\rho}} \boldsymbol{\rho}-C(\mathbf{q}, \dot{\mathbf{q}}) \dot{\mathbf{q}}]
\end{array}\right] \\
& J=\left[\begin{array}{lll}
\mathbf{0} & \mathbf{0} & \mathbf{I}
\end{array}\right]^{T}
\end{aligned}
$$

where $\mathbf{0} \in \mathbb{R}^{6 \times 6}$ and $\mathbf{I} \in \mathbb{R}^{6 \times 6}$ stands for the zero and the identity matrices, respectively, and $\mathbf{U}=\mathbf{u}_{\tau}-\mathbf{G}(\mathbf{q}) \in \mathbb{R}^{6}$.

Assuming that the parameters of the system in the vector $\boldsymbol{\gamma}=\left[\begin{array}{lll}\gamma_{1} & \ldots & \gamma_{9}\end{array}\right]^{T}=\left[\begin{array}{lllllllll}m_{r} & m_{l} & m_{p_{1}} & m_{p_{2}} & l_{l} & l_{p_{1}} & l_{p_{2}} & I_{l} & g\end{array}\right]^{T} \in \mathbb{R}^{9}$ are subjected to some degree of uncertainty, the performance of the system is degraded as a consequence. Even when the gravity acceleration is not a parameter of the system, a deviation from the nominal value is considered. The influence of parametric uncertainties is modeled as a noisy Gaussian signal with zero 


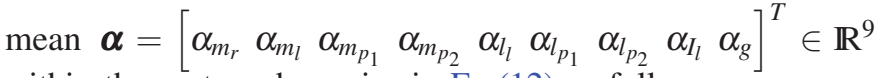
within the system dynamics in Eq.(12) as follows:

$$
\dot{\chi}^{e}=\mathbf{F}\left(\boldsymbol{\chi}^{e}, \mathbf{U}\right)+J \boldsymbol{\vartheta}+Z \boldsymbol{\alpha}
$$

The influence of the parametric deviation is weighted by $Z \in \mathbb{R}^{18 \times 9}$ that contains the partial derivative of the function $\mathbf{F}\left(\boldsymbol{\chi}^{e}, \mathbf{U}\right)$ w.r.t. the corresponding parameter, such that:

$$
Z=\left.\left[\begin{array}{llll}
\frac{\partial \mathbf{F}\left(\boldsymbol{\chi}^{e}, \mathbf{U}\right)}{\partial m_{r}} & \frac{\partial \mathbf{F}\left(\boldsymbol{\chi}^{e}, \mathbf{U}\right)}{\partial m_{l}} & \ldots & \frac{\partial \mathbf{F}\left(\boldsymbol{\chi}^{e}, \mathbf{U}\right)}{\partial g}
\end{array}\right]\right|_{\boldsymbol{\chi}^{e}, \mathbf{U}}
$$

This definition implies the computation of the partial derivatives and their evaluation at each time step and at the current $\chi^{e}$ and $\mathbf{U}$. In this regard, the dynamics of $\mathbf{q}$ and that of $\boldsymbol{\rho}$, as described in Eq.(12), do not depend on the parameters in $\gamma$ which leads to:

$$
\frac{\partial \dot{\mathbf{q}}}{\partial \boldsymbol{\gamma}}=\frac{\partial \dot{\boldsymbol{\rho}}}{\partial \boldsymbol{\gamma}}=\mathbf{0}^{\prime} \in \mathbb{R}^{6 \times 9}
$$

On the other hand, the dynamics of $\dot{\mathbf{q}}$ does not allow one to compute the partial derivatives with that easiness. The definition of $\ddot{\mathbf{q}}$ established in Eq.(9) implies the computation of the inverse of the inertia matrix $M(\mathbf{q})$ which is computed considering its adjugate, $A_{M} \in \mathbb{R}^{6 \times 6}$, and its determinant $d_{M} \in \mathbb{R}^{+}$:

$$
M(\mathbf{q})^{-1}=d_{M}^{-1} A_{M}^{T}
$$

Thus, Eq.(9) can be rewritten as follows:

$$
\ddot{\mathbf{q}}=d_{M}^{-1} A_{M}^{T} \mathbf{v}
$$

where $\mathbf{v}=\mathbf{U}+\boldsymbol{\rho}-C(\mathbf{q}, \dot{\mathbf{q}}) \dot{\mathbf{q}}$. Such substitutions ease the expression manipulation regarding the computation of $M(\mathbf{q})^{-1}$ and the derivatives within the software used. Thus, the partial derivative of $\ddot{\mathbf{q}}$ in Eq.(19) can be computed as:

$$
\frac{\partial \ddot{\mathbf{q}}}{\partial \boldsymbol{\gamma}}=\frac{\partial}{\partial \boldsymbol{\gamma}}\left(\frac{1}{d_{M}} A_{M}^{T} \mathbf{v}\right)=\frac{\partial}{\partial \boldsymbol{\gamma}}\left(\frac{1}{d_{M}} A_{M}^{T}\right) \mathbf{v}+\frac{1}{d_{\mathbf{M}}} A_{M}^{T} \frac{\partial \mathbf{v}}{\partial \boldsymbol{\gamma}}
$$

which can be expanded in the manner that:

$$
\ddot{q}_{\gamma}=\frac{\partial \ddot{\mathbf{q}}}{\partial \boldsymbol{\gamma}}=\frac{1}{d_{M}}\left[\frac{\partial A_{M}^{T}}{\partial \boldsymbol{\gamma}} \mathbf{v}-\left(\frac{1}{d_{M}} A_{M}^{T} \mathbf{v}\right) \frac{\partial d_{M}}{\partial \boldsymbol{\gamma}}\right]+\frac{1}{d_{M}} A_{M}^{T} \mathbf{v}_{\gamma}
$$

Considering Eqs.(18) and (19), one obtains:

$$
\ddot{q}_{\gamma}=d_{M}^{-1}\left(A_{M_{\gamma}}-\ddot{\mathbf{q}} \mathbf{d}_{M_{\gamma}}\right)+M(\mathbf{q})^{-1} \mathbf{v}_{\gamma}
$$

where

$$
\begin{aligned}
A_{M_{\gamma}} & =\left[\begin{array}{lllll}
\frac{\partial A_{M}^{T}}{\partial m_{r}} \mathbf{v} & \frac{\partial A_{M}^{T}}{\partial m_{l}} \mathbf{v} & \ldots & \frac{\partial A_{M}^{T}}{\partial I_{l}} \mathbf{v} & \frac{\partial A_{M}^{T}}{\partial g} \mathbf{v}
\end{array}\right] \in \mathbb{R}^{6 \times 9} \\
\mathbf{d}_{M_{\gamma}} & =\frac{\partial d_{M}}{\partial \boldsymbol{\gamma}}=\left[\begin{array}{lllll}
\frac{\partial d_{M}}{\partial m_{r}} & \frac{\partial d_{M}}{\partial m_{l}} & \ldots & \frac{\partial d_{M}}{\partial l_{l}} & \frac{\partial d_{M}}{\partial g}
\end{array}\right] \in \mathbb{R}^{1 \times 9} \\
\mathbf{v}_{\gamma} & =\frac{\partial \mathbf{v}}{\partial \boldsymbol{\gamma}}=\left[\begin{array}{lllll}
\frac{\partial \mathbf{v}}{\partial m_{r}} & \frac{\partial \mathbf{v}}{\partial m_{l}} & \ldots & \frac{\partial \mathbf{v}}{\partial I_{l}} & \frac{\partial \mathbf{v}}{\partial g}
\end{array}\right] \in \mathbb{R}^{6 \times 9}
\end{aligned}
$$

Redefining $Z$ to be:

$$
Z=\left.\left[\begin{array}{lll}
\mathbf{0}^{T} & \ddot{q}_{\gamma}^{T} & \mathbf{0}^{\prime T}
\end{array}\right]^{T}\right|_{\chi^{e}, \mathbf{U}}
$$

We found the advantage of such definition of $Z$ in the computation of the partial derivatives $A_{M_{\gamma}}, \mathbf{d}_{M_{\gamma}}$ and $\mathbf{v}_{\gamma}$ as they are straightforward to be easily manipulated within the software environment. Thus, the most relative expensive computational task to be developed at each loop is the computation of
$M(\mathbf{q})^{-1}$. Moreover, one possible solution to this issue can be to implement the definition given in Eq.(18) in a user defined function for evaluation only.

The two noise signals in Eq.(15) can be regrouped into one single vector $\boldsymbol{\omega}=\left[\begin{array}{ll}\boldsymbol{\alpha}^{T} & \boldsymbol{\vartheta}^{T}\end{array}\right]^{T} \in \mathbb{R}^{15}$ therefore, Eq.(15) can be rewritten as:

$$
\dot{\chi}^{e}=\mathbf{F}\left(\boldsymbol{\chi}^{e}, \mathbf{U}\right)+\mathscr{M} \boldsymbol{\omega}
$$

with $\mathscr{M}=\left[\begin{array}{ll}Z & J\end{array}\right] \in \mathbb{R}^{18 \times 15}$. The observation model of the system is directly expressed in a discrete domain [24]-[36] as:

$$
\mathbf{Y}_{k}^{e}=C_{k}^{e} \boldsymbol{\chi}_{k}^{e}+\boldsymbol{V}_{k}
$$

with $\mathbf{Y}_{k}^{e} \in \mathbb{R}^{12}, \boldsymbol{V}_{k}=\left[\begin{array}{llll}v_{x_{k}} & v_{z_{k}} & \ldots & v_{\dot{\beta}_{2 k}}\end{array}\right]^{T} \in \mathbb{R}^{12}$ and

$$
C_{k}^{e}=\left[\begin{array}{lll}
\mathbf{I} & \mathbf{0} & \mathbf{0} \\
\mathbf{0} & \mathbf{I} & \mathbf{0}
\end{array}\right] \in \mathbb{R}^{12 \times 18}
$$

implying that the $\mathbf{q}$ and $\dot{\mathbf{q}}$ are available but noisy since the vector $\boldsymbol{V}_{k}$ corresponds to the sensor Gaussian noise variances.

\section{Uncertainties Estimation Algorithm}

Due to the high non-linearity and couplings of the dynamics, the discretization process becomes complex and highly computationally expensive. A continuous - discrete Augmented State EKF is thus conceived. In this regard, the prediction phase is executed considering a continuous time representation of the system (Eq.(27)) meanwhile, Eq.(28) is used in the correction phase [35], [36]. Moreover, the system is considered to be observable at a given operation point (Appendix B).

The signals noises $\boldsymbol{\omega}$ and $\boldsymbol{V}_{k}$ previously introduced in Section III, stand for uncorrelated white Gaussian random processes with zero mean, i.e. $E\left[\boldsymbol{\omega}(t) \boldsymbol{V}_{k}(t)^{T}\right]=\mathbf{0}^{*} \in \mathbb{R}^{15 \times 12}$, $E[\boldsymbol{\omega}(t)]=0$ and $E\left[\boldsymbol{V}_{k}(t)\right]=0$. The process covariance matrix $Q \in \mathbb{R}^{15 \times 15}$ is characterized by $\mathscr{M}$ as:

$$
Q(t)=E\left[\boldsymbol{\omega} \boldsymbol{\omega}^{T}\right]=E\left[(\mathscr{M} \mathbf{w})(\mathscr{M} \mathbf{w})^{T}\right]=\mathscr{M} W \mathscr{M}^{T}
$$

where $W=E\left[\mathbf{w w}^{T}\right] \in \mathbb{R}^{15 \times 15}$ is a diagonal matrix containing the variances of the process noise signals. The covariance matrix of the measurement noise $R_{k}=$ $\operatorname{diag}\left\{E\left[v_{x_{k}}\right], E\left[v_{z_{k}}\right], \ldots, E\left[v_{\dot{\beta}_{1_{k}}}\right], E\left[v_{\dot{\beta}_{2_{k}}}\right]\right\} \in \mathbb{R}^{12 \times 12}$ is defined by the variances of the noisy sensors.

The prediction phase of the AS-EKF is thus described by:

$$
\begin{aligned}
\dot{\hat{\chi}}^{e}(t) & =\mathbf{F}\left(\hat{\chi}^{e}, \mathbf{U}\right) \\
\dot{P}(t) & =F_{\chi^{e}}\left(\hat{\chi}^{e}, \mathbf{U}\right) P(t)+P(t) F_{\chi^{e}}^{T}\left(\hat{\boldsymbol{\chi}}^{e}, \mathbf{U}\right)+Q(t)
\end{aligned}
$$

with

$$
F_{\chi^{e}}\left(\hat{\boldsymbol{\chi}}^{e}, \mathbf{U}\right)=\left.\frac{\partial \mathbf{F}\left(\boldsymbol{\chi}^{e}, \mathbf{U}\right)}{\partial \boldsymbol{\chi}^{e}}\right|_{\chi^{e}, \mathbf{U}} \in \mathbb{R}^{18 \times 18}
$$

computed by the same procedure used to define $\mathbf{Z}$. Notice that $\hat{\chi}^{e}=\hat{\chi}^{e}(t)$ and $\mathbf{U}=\mathbf{U}(t)$.

The set of differential equations in Eqs.(31) and (32) is solved via numerical methods to find $\hat{\chi}^{e}(t)$ and $P(t)$ [36]. For the first iteration, the initial values of $\hat{\chi}^{e}(t)$ and $P(t)$ are given as $\hat{\chi}_{0}^{e}$ and $P_{0}$, respectively.

In order to adopt the values obtained during the prediction phase 


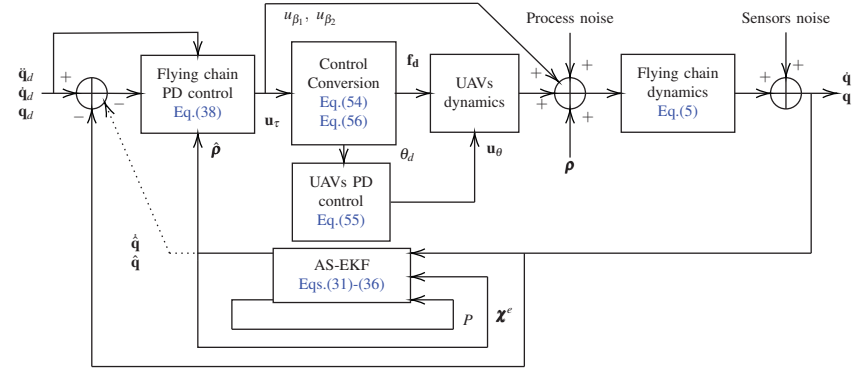

Figure 3: Control block diagram

$\hat{\chi}_{k \mid k-1}^{e}=\hat{\chi}^{e}\left(t_{k}\right)$ and $P_{k \mid k-1}=P\left(t_{k}\right)$ for ulterior computations. The correction phase is given by the set of equations:

$$
\begin{aligned}
K_{k} & =P_{k \mid k-1} C_{k}^{e T}\left(C_{k}^{e} P_{k \mid k-1} C_{k}^{e T}+R_{k}\right)^{-1} \\
\hat{\boldsymbol{\chi}}_{k \mid k}^{e} & =\hat{\boldsymbol{\chi}}_{k \mid k-1}^{e}+K_{k}\left(\mathbf{Y}_{k}^{e}-C_{k}^{e} \hat{\boldsymbol{\chi}}_{k \mid k-1}^{e}\right) \\
P_{k \mid k} & =\left(\mathbf{I}^{\prime}-K_{k} C_{k}^{e}\right) P_{k \mid k-1}
\end{aligned}
$$

where $\mathbf{I}^{\prime} \in \mathbb{R}^{18 \times 18}$ is the identity matrix. Thus, the estimation of the disturbances is taken from the estimation:

$$
\hat{\boldsymbol{\chi}}_{k \mid k}^{e}=\left[\begin{array}{lll}
\hat{\mathbf{q}}_{k \mid k}^{T} & \dot{\mathbf{q}}_{k \mid k}^{T} & \hat{\boldsymbol{\rho}}_{k \mid k}^{T}
\end{array}\right]^{T}
$$

The sub-index $k \mid k$ is omitted from here until the end of the document for sake of simplicity in the expressions.

\section{CONTROL}

The control of the overall ML-UAS implies two different control loops: one related to the flying chain and the second one referred to the control of the aircrafts (inner control loop). For a proper identification, a block diagram is presented in Fig 3 . The estimation of the disturbances is added to the control loop as shown meanwhile, the estimation of the states is used to close the loop when a given sensor fails (doted arrow).

A PD control law with disturbances compensation:

$$
\mathbf{u}_{\tau}=K_{P} \mathbf{e}+K_{V} \dot{\mathbf{e}}+M\left(\mathbf{q}_{d}\right) \ddot{\mathbf{q}}_{d}+C\left(\mathbf{q}_{d}, \dot{\mathbf{q}}_{d}\right) \dot{\mathbf{q}}_{d}+\mathbf{G}\left(\mathbf{q}_{d}\right)-\hat{\boldsymbol{\rho}}
$$

where $K_{P}>0 \in \mathbb{R}^{6 \times 6}$ and $K_{V}>0 \in \mathbb{R}^{6 \times 6}$ are diagonal matrices that stand for the proportional and derivative gains, respectively, is applied to the system. The vectors $\mathbf{e}=\mathbf{q}_{d}-\mathbf{q} \in \mathbb{R}^{6}$ and $\dot{\mathbf{e}}=\dot{\mathbf{q}}_{d}-\dot{\mathbf{q}} \in \mathbb{R}^{6}$ depend on the desired position $\mathbf{q}_{d}$ and the desired velocity $\dot{\mathbf{q}}_{d}$ and correspond to the position and velocity errors, respectively.

The stability of the close loop system is studied via a Lyapunov based-method considering the properties of the system exposed in Appendix A.

\section{A. Lyapunov Stability Analysis}

By considering the control law in Eq.(38), taking into account the model of the system introduced in Eq.(5) and assuming $\hat{\boldsymbol{\rho}} \approx \boldsymbol{\rho}$, the closed loop equation can be expressed as:

$$
\frac{d}{d t}\left[\begin{array}{c}
\mathbf{e} \\
\dot{\mathbf{e}}
\end{array}\right]=\left[\begin{array}{c}
\dot{\mathbf{e}} \\
M(\mathbf{q})^{-1}\left[-K_{P} \mathbf{e}-K_{V} \dot{\mathbf{e}}-C(\mathbf{q}, \dot{\mathbf{q}}) \dot{\mathbf{e}}-\mathbf{h}(\mathbf{e}, \dot{\mathbf{e}})\right]
\end{array}\right]
$$

The equilibrium $\left[\mathbf{e}_{e}^{T} \dot{\mathbf{e}}_{e}^{T}\right]^{T} \in \mathbb{R}^{12}$ of Eq.(39) is located at $\dot{\mathbf{e}}_{e}=\mathbf{0}^{\star}$ although the vector $\mathbf{e}_{e}$ can take different values. In order to ensure globally asymptotically stability of the system, the uniqueness of the equilibrium point is mandatory. In this regard and according to [37], the matrix $K_{P}$ can be selected such that

$$
\lambda_{\text {min }}\left(K_{P}\right)>k_{g}+k_{M}\left\|\ddot{\mathbf{q}}_{d}\right\|_{\text {max }}+k_{C_{2}}\left\|\dot{\mathbf{q}}_{d}\right\|_{\text {max }}^{2}
$$

The constants $k_{g}, k_{M}$ and $k_{C_{1}}$ are defined in the Appendix A. To perform the stability analysis, let it exist a constant $\varepsilon \in \mathbb{R}^{+}$ such that it defines the matrices $K_{V}$ and $K_{P}$ as follows:

$$
\begin{aligned}
& \lambda_{\text {Max }}\left(K_{V}\right) \geq \lambda_{\min }\left(K_{V}\right)>k_{h_{1}}+\varepsilon b \\
& \lambda_{\text {Max }}\left(K_{P}\right) \geq \lambda_{\min }\left(K_{P}\right)>\frac{\left(2 \varepsilon a+k_{h_{2}}\right)^{2}}{4 \varepsilon\left[\lambda_{\min }\left(K_{V}\right)-k_{h_{1}}-\varepsilon b\right]}+k_{h_{2}} \\
& \lambda_{\text {Max }}\left(K_{P}\right) \geq \lambda_{\min }\left(K_{P}\right)>\varepsilon^{2} \frac{\lambda_{\text {Max }}^{2}[M(\mathbf{q})]}{\lambda_{\min }[M(\mathbf{q})]}
\end{aligned}
$$

with $k_{h_{1}}$ and $k_{h_{2}}$ introduced in Appendix $\mathrm{A}$ and

$$
\begin{aligned}
a & =\frac{1}{2}\left[\lambda_{\text {Max }}\left(K_{V}\right)+k_{C_{1}}\left\|\dot{\mathbf{q}}_{d}\right\|_{\text {max }}+k_{h_{1}}\right] \in \mathbb{R}^{+} \\
b & =\lambda_{\text {Max }}[M(\mathbf{q})]+\sqrt{6} k_{C_{1}} \in \mathbb{R}^{+}
\end{aligned}
$$

Let the Lyapunov candidate function $V(t, \mathbf{e}, \dot{\mathbf{e}}) \in \mathbb{R}$ be

$$
V=\frac{1}{2} \dot{\mathbf{e}}^{T} M(\mathbf{q}) \dot{\mathbf{e}}+\frac{1}{2} \mathbf{e}^{T} K_{P} \mathbf{e}+\varepsilon \mathbf{f}_{t h}(\mathbf{e})^{T} M(\mathbf{q}) \dot{\mathbf{e}}
$$

with $\mathbf{f}_{t h}(\mathbf{e})$ the hyperbolic tangent function (see Appendix A). Since $M(\mathbf{q})$ and $K_{P}$ are positive definite matrices by definition and as $K_{P}$ satisfies Eq.(41), it is trivial to conclude that

$$
\begin{aligned}
\dot{\mathbf{e}}^{T} M(\mathbf{q}) \dot{\mathbf{e}} & \geq \lambda_{\min }[M(\mathbf{q})]\|\dot{\mathbf{e}}\|^{2} \\
\mathbf{e}^{T} K_{P} \mathbf{e} & \geq \lambda_{\min }\left(K_{P}\right)\|\mathbf{e}\|^{2}
\end{aligned}
$$

$\forall \mathbf{q}, \mathbf{e}, \dot{\mathbf{e}} \in \mathbb{R}^{6}$. Moreover, given the properties of the system in Appendix A, it is possible to obtain that:

$$
\varepsilon \mathbf{f}_{t h}(\mathbf{e})^{T} M(\mathbf{q}) \dot{\mathbf{e}} \geq-\varepsilon \lambda_{\operatorname{Max}}[M(\mathbf{q})]\|\mathbf{e}\|\|\dot{\mathbf{e}}\|
$$

Thus, $V(t, \mathbf{e}, \dot{\mathbf{e}})$ satisfies:

$$
V \geq \frac{1}{2}\left[\begin{array}{l}
\|\mathbf{e}\| \\
\|\dot{\mathbf{e}}\|
\end{array}\right]^{T}\left[\begin{array}{cc}
\lambda_{\min }\left(K_{P}\right) & -\varepsilon \lambda_{\operatorname{Max}}[M(\mathbf{q})] \\
-\varepsilon \lambda_{\operatorname{Max}}[M(\mathbf{q})] & \lambda_{\min }[M(\mathbf{q})]
\end{array}\right]\left[\begin{array}{l}
\|\mathbf{e}\| \\
\|\dot{\mathbf{e}}\|
\end{array}\right]
$$

Concluding that $V(t, \mathbf{e}, \dot{\mathbf{e}})$ is a radially unbounded positive definite function.

The time derivative of the Lyapunov candidate function $\dot{V}(t, \mathbf{e}, \dot{\mathbf{e}}) \in \mathbb{R}$ in Eq.(44) along the trajectories of the closed loop system can be expressed as:

$$
\begin{aligned}
\dot{V}= & -\dot{\mathbf{e}}^{T} K_{V} \dot{\mathbf{e}}-\varepsilon \mathbf{f}_{t h}(\mathbf{e})^{T} K_{P} \mathbf{e}+\varepsilon \mathbf{f}_{t h}(\mathbf{e})^{T}\left[C(\mathbf{q}, \dot{\mathbf{q}})-K_{V}\right] \dot{\mathbf{e}}- \\
& \dot{\mathbf{e}}^{T} \mathbf{h}(\mathbf{e}, \dot{\mathbf{e}})-\varepsilon \mathbf{f}_{t h}(\mathbf{e})^{T} \mathbf{h}(\mathbf{e}, \dot{\mathbf{e}})+\varepsilon \dot{\mathbf{f}}_{t h}(\mathbf{e})^{T} M(\mathbf{q}) \dot{\mathbf{e}}
\end{aligned}
$$

From Appendix A, it is possible to establish that:

$$
\begin{aligned}
-\dot{\mathbf{e}}^{T} K_{V} \dot{\mathbf{e}} & \leq-\lambda_{\min }\left(K_{V}\right)\|\dot{\mathbf{e}}\|^{2} \\
\varepsilon \dot{\mathbf{f}}_{t h}(\mathbf{e})^{T} M(\mathbf{q}) \dot{\mathbf{e}} & \leq \varepsilon \lambda_{\operatorname{Max}}[M(\mathbf{q})]\|\dot{\mathbf{e}}\|^{2} \\
-\varepsilon \mathbf{f}_{t h}(\mathbf{e})^{T} K_{P} \mathbf{e} & \leq-\varepsilon \lambda_{\min }\left(K_{P}\right)\left\|\mathbf{f}_{t h}(\mathbf{e})\right\|^{2} \\
\varepsilon \mathbf{f}_{t h}(\mathbf{e})^{T} K_{V} \dot{\mathbf{e}} & \leq \varepsilon \lambda_{\operatorname{Max}}\left(K_{V}\right)\|\dot{\mathbf{e}}\|\left\|\mathbf{f}_{t h}(\mathbf{e})\right\| \\
\varepsilon \mathbf{f}_{t h}(\mathbf{e})^{T} C(\mathbf{q}, \dot{\mathbf{q}})^{T} \dot{\mathbf{e}} & \leq \varepsilon k_{C_{1}}\left(\left\|\dot{\mathbf{q}}_{d}\right\|_{\max }\|\dot{\mathbf{e}}\|\left\|\mathbf{f}_{t h}(\mathbf{e})\right\|+\sqrt{6}\|\dot{\mathbf{e}}\|^{2}\right) \\
-\dot{\mathbf{e}}^{T} \mathbf{h}(\mathbf{e}, \dot{\mathbf{e}}) & \leq k_{h_{1}}\|\dot{\mathbf{e}}\|^{2}+k_{h_{2}}\|\dot{\mathbf{e}}\|\left\|\mathbf{f}_{t h}(\mathbf{e})\right\| \\
-\varepsilon \mathbf{f}_{t h}(\mathbf{e})^{T} \mathbf{h}(\mathbf{e}, \dot{\mathbf{e}}) & \leq \varepsilon k_{h_{1}}\|\dot{\mathbf{e}}\|\left\|\mathbf{f}_{t h}(\mathbf{e})\right\|+\varepsilon k_{h_{2}}\left\|\mathbf{f}_{t h}(\mathbf{e})\right\|^{2}
\end{aligned}
$$


$\dot{V}(t, \mathbf{e}, \dot{\mathbf{e}})$ in Eq.(48) satisfies the inequality

$$
\dot{V} \leq-\varepsilon\left[\begin{array}{c}
\left\|\mathbf{f}_{t h}(\mathbf{e})\right\| \\
\|\mathbf{e}\|
\end{array}\right]^{T} R_{M}(\varepsilon)\left[\begin{array}{c}
\left\|\mathbf{f}_{t h}(\mathbf{e})\right\| \\
\|\mathbf{e}\|
\end{array}\right]
$$

with $R_{M}(\varepsilon) \in \mathbb{R}^{2 \times 2}$ being

$$
R_{M}(\varepsilon)=\left[\begin{array}{cc}
\lambda_{\min }\left(K_{P}\right)-k_{h_{2}} & -\left(a+\frac{k_{h_{2}}}{2 \varepsilon}\right) \\
-\left(a+\frac{k_{h_{2}}}{2 \varepsilon}\right) & \frac{1}{\varepsilon}\left[\lambda_{\min }\left(K_{V}\right)-k_{h_{1}}\right]-b
\end{array}\right]
$$

thus $\dot{V}(t, \mathbf{e}, \dot{\mathbf{e}})$ is a global definite negative function if the matrix $R_{M}(\varepsilon)$ is definite positive which is guaranteed if the first element of the matrix and its determinant are strictly greater than zero. In this regard, it is sufficient and enough that:

$$
\begin{aligned}
& \lambda_{\text {min }}\left(K_{P}\right)>\frac{\left(2 \varepsilon a+k_{h_{2}}\right)^{2}}{4 \varepsilon\left[\lambda_{\text {min }}\left(K_{V}\right)-k_{h_{1}}-\varepsilon b\right]}+k_{h_{2}} \\
& \lambda_{\text {min }}\left(K_{V}\right)>k_{h_{1}}+\varepsilon b
\end{aligned}
$$

With a proper selection of a constant $\varepsilon$ the matrices $K_{P}$ and $K_{V}$ can be computed such that $V(t, \mathbf{e}, \dot{\mathbf{e}})$ is a radially unbounded positive definite function and $\dot{V}(t, \mathbf{e}, \dot{\mathbf{e}})$ is a globally negative definite function, concluding that the $V(t, \mathbf{e}, \dot{\mathbf{e}})$ is a strict Lyapunov function thus the system possesses globally asymptotically stability [37].

\section{B. Inner Control Loop}

The desired thrust and attitude of the vehicles are computed according to Eqs.(8) and (38). Having in mind the overactuation of the ML-UAS is over-actuated since it has 6 DOFs and 8 real control inputs $\left(f_{i}, \theta_{i_{d}}\right.$ and $\left.t_{\beta_{j}}\right)$; the desired orientation angle of the UAVs is established to be $\theta_{1_{d}}=\theta_{2_{d}}=\theta_{3_{d}}=\theta_{d}$, to avoid the prescribed condition. This assumption can be considered in Eq.(8), leading to:

$$
\mathbf{u}_{\tau}=\left[\begin{array}{c}
\left(f_{1}+f_{2}+f_{3}\right) S_{\theta_{d}} \\
\left(f_{1}+f_{2}+f_{3}\right) C_{\theta_{d}} \\
\frac{l_{l}}{2}\left(f_{1}-f_{2}\right) C_{\Theta_{1}-\theta_{d}} \\
\frac{l_{l}}{2}\left(f_{2}-f_{3}\right) C_{\Theta_{2}-\theta_{d}} \\
t_{\beta_{1}} \\
t_{\beta_{2}}
\end{array}\right]
$$

Thus, the desired orientation of the UAVs is defined as:

$$
\theta_{d}=\tan ^{-1}\left(u_{x} / u_{z}\right)
$$

Notice that $u_{z}>0$ as it tends to the total desired thrust.

The $i^{t h}$ vehicle is driven to $\theta_{d}$ via a PD controller of the form:

$$
u_{\theta_{i}}=K_{p_{\theta}} e_{\theta_{i}}+K_{v_{\theta}} \dot{e}_{\theta_{i}}
$$

with $K_{p_{\theta}}, K_{v_{\theta}}>0 \in \mathbb{R}$ the proportional and derivative gains and $e_{\theta_{i}}, \quad \dot{e}_{\theta_{i}} \in \mathbb{R}$ the position and velocity errors of the $i^{\text {th }}$ vehicle. The stability proof is available at [38].

The total thrust exerted by the vehicles is computed such that:

$$
\mathbf{f}_{\mathbf{d}}=\left[\begin{array}{l}
f_{1} \\
f_{2} \\
f_{3}
\end{array}\right]=\frac{1}{3}\left[\begin{array}{ccc}
1 & 2 & 1 \\
1 & -1 & 1 \\
1 & -1 & -2
\end{array}\right]\left[\begin{array}{c}
\sqrt{u_{x}^{2}+u_{z}^{2}} \\
\frac{2}{l_{l} C_{\Theta_{1}-\theta_{d}}} u_{\Theta_{1}} \\
\frac{2}{l_{l} C_{\Theta_{2}-\theta_{d}}} u_{\Theta_{2}}
\end{array}\right] \in \mathbb{R}^{3}
$$

To validate the proposal, a detailed simulation was conducted within a close-to-reality scenario.

\section{NUMERICAL Simulations}

The current section is devoted to validate the effectiveness of the AS-EKF and the proposed control strategy. In this regard, the aerial robotic system was intended to follow a prescribed time-dependent trajectory $\mathbf{q}_{d}$ given as:

$$
\mathbf{q}_{d}=\left[\begin{array}{c}
-\sin \left(4 \pi \frac{t}{100}\right)[\mathrm{m}] \\
3+\cos \left(4 \pi \frac{t}{100}\right)[\mathrm{m}] \\
\frac{1}{4} \cos \left(4 \pi \frac{t}{100}\right)[\mathrm{rad}] \\
-0.6458[\mathrm{rad}] \\
0.4363[\mathrm{rad}] \\
-\frac{1}{2} \sin \left(4 \pi \frac{t}{100}\right)[\mathrm{rad}]
\end{array}\right]
$$

The vectors $\dot{\mathbf{q}}_{d}$ and $\ddot{\mathbf{q}}_{d}$ were computed by differentiation of Eq.(57) w.r.t. time. Moreover, three sensors were considered to fail during the simulation. The $x, \dot{\Theta}_{1}$ and $\beta_{2}$ sensors faults occur at $t=29, t=55$ and $t=78$, respectively. The total simulation time was $100 \mathrm{~s}$ with a sampling time $d t=0.01$.

The properties of the system parameters as well as those of the sensors and the disturbances used for simulation are presented in Table I. In this matter, the disturbances vector was defined as $\boldsymbol{\rho}=\left[\begin{array}{llll}1.52 \cos \left(\frac{t \pi}{55}\right) & -0.55 \cos \left(\frac{t \pi}{33}\right)-0.5 \sin \left(\frac{t \pi}{45}\right) & 0.75\end{array}\right]^{T}$ with the corresponding units.

The initial conditions of the overall system were all set to 0 ,

\begin{tabular}{|c|c|c|c|}
\hline \multicolumn{4}{|c|}{ Parameters properties } \\
\hline Parameter & \multicolumn{2}{|c|}{ Nominal value } & Variance \\
\hline$m_{r}$ & \multicolumn{2}{|c|}{$0.653 \mathrm{~kg}$} & 0.0011111 \\
\hline$m_{l}$ & \multicolumn{2}{|c|}{$0.10 \mathrm{~kg}$} & 0.0000693 \\
\hline$m_{p_{1}}$ & \multicolumn{2}{|c|}{$0.272 \mathrm{~kg}$} & 0.0002775 \\
\hline$m_{p_{2}}$ & \multicolumn{2}{|c|}{$0.383 \mathrm{~kg}$} & 0.0002775 \\
\hline$l_{l}$ & \multicolumn{2}{|c|}{$1.25 \mathrm{~m}$} & 0.000544 \\
\hline$l_{p_{1}}, l_{p_{2}}$ & \multicolumn{2}{|c|}{$0.5 \mathrm{~m}$} & 0.0002775 \\
\hline$I_{l}$ & \multicolumn{2}{|c|}{$0.172 \mathrm{~kg} \mathrm{~m}^{2}$} & 0.000100 \\
\hline$g$ & \multicolumn{2}{|c|}{$9.81 \mathrm{~ms}^{-2}$} & 0.004900 \\
\hline \multicolumn{4}{|c|}{ Sensors properties } \\
\hline Sensor & Variance & Sensor & Variance \\
\hline$x$ & $2.3 \times 10^{-4}$ & $\dot{x}$ & $5 \times 10^{-4}$ \\
\hline$z$ & $3.5 \times 10^{-4}$ & $\dot{z}$ & $6.5 \times 10^{-4}$ \\
\hline$\Theta_{1}, \Theta_{2}$ & $2.6 \times 10^{-4}$ & $\dot{\Theta}_{1}, \dot{\Theta}_{2}$ & $1.7 \times 10^{-4}$ \\
\hline$\beta_{1}, \beta_{2}$ & $2.3 \times 10^{-4}$ & $\dot{\beta}_{1}, \dot{\beta}_{2}$ & $5.1 \times 10^{-5}$ \\
\hline \multicolumn{4}{|c|}{ Disturbances properties } \\
\hline \multicolumn{2}{|c|}{ Disturbance } & \multicolumn{2}{|c|}{ Variance } \\
\hline \multicolumn{2}{|c|}{$\rho_{x}, \rho_{z}$} & \multicolumn{2}{|c|}{$3 \times 10^{-4}$} \\
\hline \multicolumn{2}{|c|}{$\rho_{\Theta_{1}}, \rho_{\Theta_{2}}$} & \multicolumn{2}{|c|}{$1 \times 10^{-4}$} \\
\hline \multicolumn{2}{|c|}{$\rho_{\beta_{1}}, \rho_{\beta_{2}}$} & \multicolumn{2}{|c|}{$7 \times 10^{-5}$} \\
\hline
\end{tabular}
meanwhile, the covariance matrix $P$ was initialized as:

$$
P_{0}=0.01\left[\begin{array}{ccccccc}
\mathbf{I}^{\dagger} & & & \mathbf{0}^{\dagger} & & & \\
& 10 & 10 & 10 & 10 & 1 & 1 \\
& 10 & 500 & 100 & 100 & 1 & 1 \\
& 10 & 100 & 250 & 100 & 1 & 0 \\
\mathbf{0}^{\dagger} & 10 & 100 & 100 & 250 & 0 & 1 \\
& 1 & 1 & 1 & 0 & 3 & 0 \\
& 1 & 1 & 0 & 1 & 0 & 3
\end{array}\right]
$$

where $\mathbf{I}^{\dagger} \in \mathbb{R}^{12 \times 12}$ and $\mathbf{0}^{\dagger} \in \mathbb{R}^{6 \times 12}$ are the identity and zero matrices, respectively.

Finally, the gain matrices in Eq.(38), were chosen to be $K_{P}=\operatorname{diag}\{8,10,19,19,7,7\}$ and

Table I: Simulation parameters 


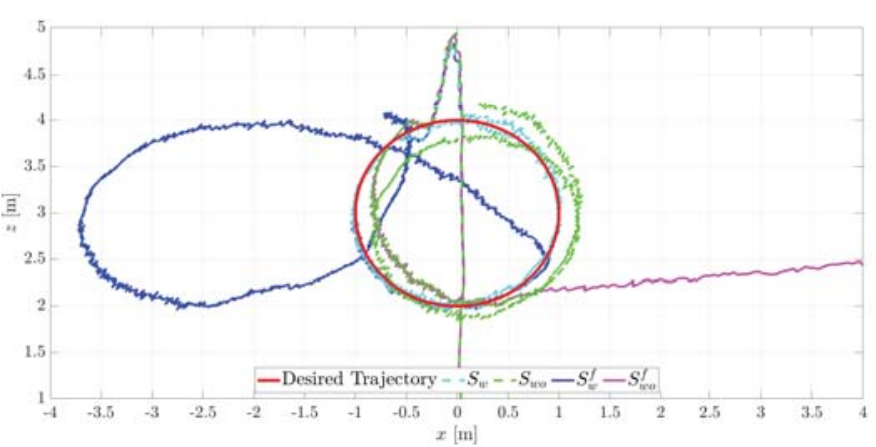

Figure 4: Translational behavior of the ML-UAS.
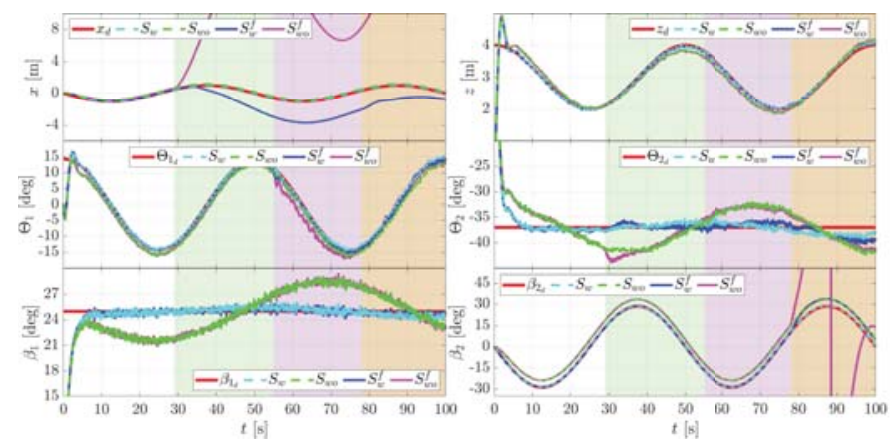

Figure 5: Position and pose evolution of the ML-UAS

$K_{V}=\operatorname{diag}\{8,15,25,25,12,12\}$. The gains in Eq.(55) were set to $K_{p_{\theta}}=7$ and $K_{v_{\theta}}=10$.

\section{Simulation Results}

During the study conducted, we simulated the system with and without the AS-EKF. The corresponding results are label as " $S_{w}$ " and " $S_{w o}$ ", respectively. In this vein, we added sensor faults to both systems to consider a more realistic scenario. $" S_{w}^{f, "}$ and " $S_{w o}^{f}$ " are the corresponding labels for the cited cases. The translational behavior of the flying system under the prescribed simulation conditions, alongside the desired trajectory, is depicted in Fig. 4.

The system without the AS-EKF clearly presents a deviation from the desired trajectory in comparison with the system where the estimation strategy is implemented. In the presence of sensors faults, the Kalman Filter provides enough information to keep the system performing (relatively) near the desired operational profile which does not occur in the $S_{w o}^{f}$ case. In this regard, Figs. 5 and 6 provide a comparison of the written herein. The background color changes stand for sensor faults. With base on Figs. 5 and 6, the implementation of the AS-EKF improves the performance of the system nevertheless, the usage of several sensors and data fusion techniques can boost the performance of the system specially when subjected to sensors failures. It is worth to mention that the estimation strategy herein is devoted to the rejection of model uncertainties and external disturbances however closing the loop with the states estimation computed by the AS-EKF could be used as a last safety measure to prevent a worst system response as shown. From Fig. 6, one can relate the presence of noise in the velocity estimation to the failure of the $\dot{\Theta}_{1}$ sensor which affects the
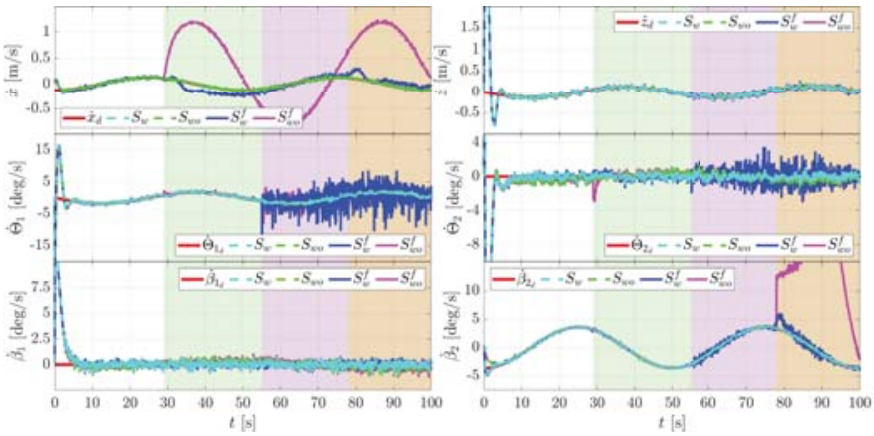

Figure 6: Velocities of the ML-UAS.

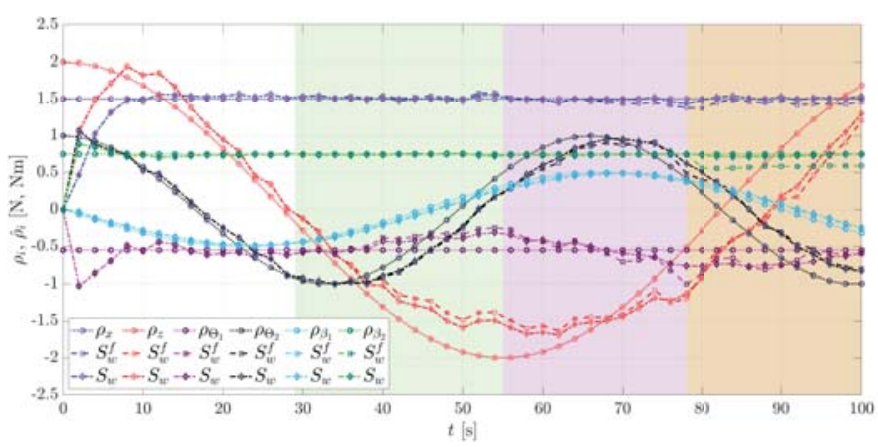

Figure 7: Disturbances estimation.

orientation of the link as well (Fig. 5). In this matter, the performance of the system when the estimation is used to close the loop differs from that of the system without the EKF in which the error is evidently greater.

Concerning the disturbances estimation and the corresponding errors, Figs. 7 and 8 depicts, respectively, the results obtained during the simulation. Notice that only few point of the available data were considered due to the highly noisy signals. According to Figs. 7 and 8, the estimation strategy provides an acceptable computation of time-independent and slow timevarying disturbances, as the parameters uncertainties. The results suggest that $\rho_{z}$ and $\rho_{\beta_{1}}$ are estimated with less accuracy as their dynamics are relatively fast in comparison with the others. One must notice that the lose of sensors has a significant impact in the estimation over $\rho_{x}, \rho_{z}, \rho_{\Theta_{1}}$ and $\rho_{\Theta_{2}}$ (for instance) as less information is available to develop the estimation properly in addition with the highly coupled dynamics.

On matters of aircrafts performance, the results are depicted in Fig. 9. The UAVs are observed to perform within the operational range of attitude and exerted thrust even when the fault of the $x$ sensor makes $\theta_{d}$ to oscillate. The thrust is also affected by the lose of sensors yet the vehicles are capable of keeping the system in track.

A video regarding the results of a detailed simulation of different scenarios is available at https://youtu.be/Nx01w0_taoM.

\section{COnCluding Remarks}

The dynamics of a novel multi-link unmanned aerial system for multiple payload transportation was obtained via the EulerLagrange formalism. Based on the non linear and the highly coupled dynamics and under the assumption of the existence of 


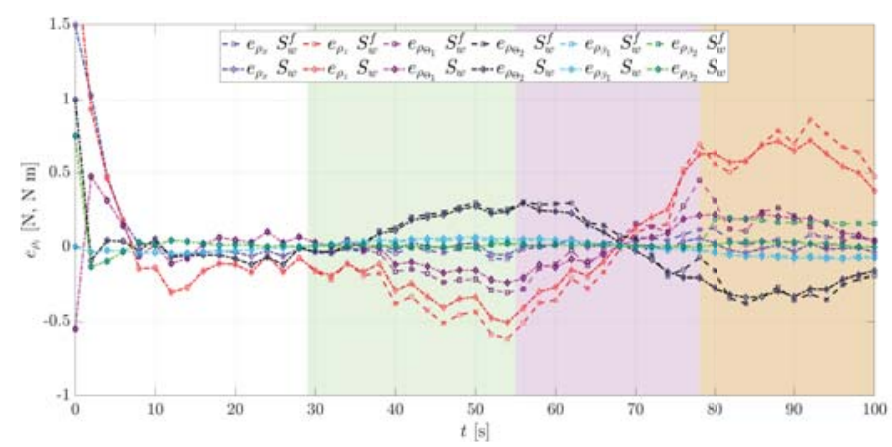

Figure 8: Disturbances estimation errors

parametric uncertainties and external unmodeled disturbances, an Augmented State Extended Kalman Filter was conceived to overcome such issues that degrade the performance of the system in order to accomplish the trajectory tracking goal. The Filter characterization takes into consideration the systems parameters uncertainties and it was proved to be efficient via close-to-reality simulations in which, the estimation was observed to be accurate enough to overcome the issues produced by the uncertainties of the systems parameters and the external disturbances. In matters of sensors fault the estimation strategy allowed to keep the system in operation closer to the desired trajectory than in the case where any information was used to close the loop.

Robust navigation strategies which take into consideration worst-case scenarios are left for upcoming studies, in this regard, different disturbance estimators are also considered to be applied over the multi-link system to compare their performance in those matters concerned to disturbances rejection.

The extension of the theory inhere introduced to a 3 dimensional space and substituting the quadrotors for convertible VTOL vehicles are left for future works as well as the practical implementation of the approach.

\section{APPENDIX A}

\section{PROPERTIES OF THE SYSTEM}

According to [37], the elements that compose the dynamical model introduced in Eq.(5) possess a set of properties which are presented in this appendix.

1) The inertia matrix $M(\mathbf{q})$ is a symmetric positive definite matrix that satisfies the following:

a) $M(\mathbf{q}) \geq \vartheta \mathbf{I} \forall \mathbf{q} \in \mathbb{R}^{6}$ with $\vartheta \in \mathbb{R}^{+}$.

b) $\lambda_{\operatorname{Max}}[M(\mathbf{q})] \leq \varsigma \forall \mathbf{q} \in \mathbb{R}^{6}$ with $\varsigma \in \mathbb{R}^{+}$

c) $\|M(\mathbf{x}) \mathbf{z}-M(\mathbf{y}) \mathbf{z}\| \leq k_{M}\|\mathbf{x}-\mathbf{y}\|\|\mathbf{z}\| \forall \mathbf{x}, \mathbf{y}, \mathbf{z} \in \mathbb{R}^{6}$ and $k_{M} \in \mathbb{R}^{+}$

d) $\|M(\mathbf{x}) \mathbf{y}\| \leq k_{M}^{\prime}\|\mathbf{y}\| \forall \mathbf{x}, \mathbf{y} \in \mathbb{R}^{6}$ and $k_{M}^{\prime} \in \mathbb{R}^{+}$

2) The centripetal effects and Coriolis terms matrix $C(\mathbf{q}, \dot{\mathbf{q}})$ can be non-unique nevertheless the vector $C(\mathbf{q}, \dot{\mathbf{q}}) \dot{\mathbf{q}}$ is unique. Additionally, this matrix satisfies the following properties:

a) $C\left(\mathbf{q}, \mathbf{0}^{\star}\right)=\mathbf{0} \forall \mathbf{q} \in \mathbb{R}^{6}$.

b) $\forall \mathbf{q}, \mathbf{x}, \mathbf{y} \in \mathbb{R}^{6},\|C(\mathbf{q}, \mathbf{x}) \mathbf{y}\| \leq k_{C_{1}}\|\mathbf{x}\|\|\mathbf{y}\|$ where $k_{C_{1}} \in \mathbb{R}^{+}$

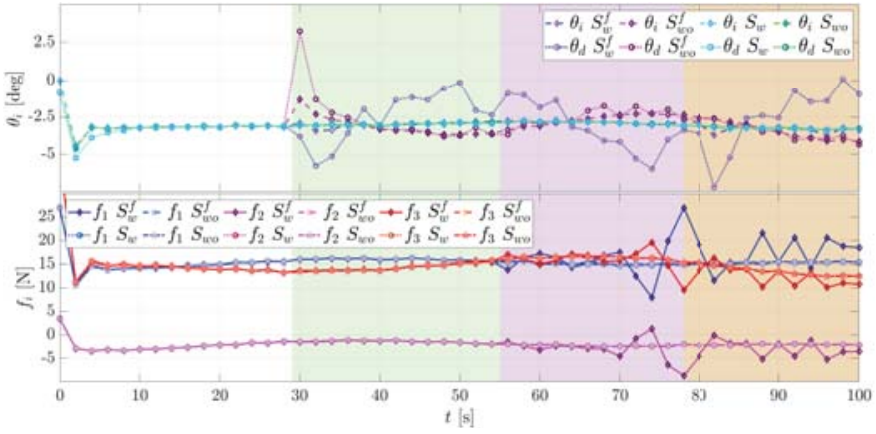

Figure 9: Aircrafts performance

c) $\forall \mathbf{v}, \mathbf{w}, \mathbf{x}, \mathbf{y}, \mathbf{z} \in \mathbb{R}^{6}$ and $k_{C_{1}}, k_{C_{2}} \in \mathbb{R}^{+}$, it holds that $\|C(\mathbf{x}, \mathbf{z}) \mathbf{w}-C(\mathbf{y}, \mathbf{v}) \mathbf{w}\| \leq k_{C_{1}}\|\mathbf{z}-\mathbf{v}\|\|\mathbf{w}\|+$ $k_{C_{2}}\|\mathbf{x}-\mathbf{y}\|\|\mathbf{w}\|\|\mathbf{z}\|$

3) The gravitational terms vector $\mathbf{G}(\mathbf{q})$ holds the properties listed next.

a) $\forall \tau_{t} \in \mathbb{R}^{+}, \int_{0}^{\tau_{t}} \mathbf{G}(\mathbf{q})^{T} \dot{\mathbf{q}} d t=\mathscr{U}\left(\mathbf{q}\left(\tau_{t}\right)\right)-\mathscr{U}(\mathbf{q}(0))$

b) $\forall \tau_{t} \in \mathbb{R}^{+}$and $k_{U}=\min _{\mathbf{q}}[\mathscr{U}(\mathbf{q})] \in \mathbb{R}^{+}$, $\int_{0}^{\tau_{t}} \mathbf{G}(\mathbf{q})^{T} \dot{\mathbf{q}} d t+\mathscr{U}(\mathbf{q}(0)) \geq k_{U}$.

c) $\forall \mathbf{x}, \mathbf{y} \in \mathbb{R}^{6},\|\mathbf{G}(\mathbf{x})-\mathbf{G}(\mathbf{y})\| \leq k_{g}\|\mathbf{x}-\mathbf{y}\|$ with

$$
k_{g} \geq\left\|\frac{\partial \mathbf{G}(\mathbf{q})}{\partial \mathbf{q}}\right\| \geq \lambda_{\operatorname{Max}}\left[\frac{\partial \mathbf{G}(\mathbf{q})}{\partial \mathbf{q}}\right] \in \mathbb{R}^{+}
$$

d) $\|\mathbf{G}(\mathbf{q})\| \leq k^{\prime} \forall \mathbf{q}$ with $k^{\prime} \in \mathbb{R}^{+}$.

4) The residual dynamics $\mathbf{h}(\mathbf{e}, \dot{\mathbf{e}}) \in \mathbb{R}^{6}$ is a vector associated to the dynamics of the robot and the position and velocity errors defined as:

$$
\begin{aligned}
\mathbf{h}(\mathbf{e}, \dot{\mathbf{e}})= & {\left[M\left(\mathbf{q}_{d}\right)-M\left(\mathbf{q}_{d}-\mathbf{e}\right)\right] \ddot{\mathbf{q}}_{d}+\mathbf{G}\left(\mathbf{q}_{d}\right)-\mathbf{G}\left(\mathbf{q}_{d}-\mathbf{e}\right) } \\
& +\left[C\left(\mathbf{q}_{d}, \dot{\mathbf{q}}_{d}\right)-C\left(\mathbf{q}_{d}-\mathbf{e}, \dot{\mathbf{q}}_{d}-\dot{\mathbf{e}}\right)\right] \dot{\mathbf{q}}_{d}
\end{aligned}
$$

Such vector satisfies that $\forall \mathbf{e}, \dot{\mathbf{e}} \in \mathbb{R}^{6}$ and $k_{h_{1}}, k_{h_{2}} \in \mathbb{R}^{+}$, $\|\mathbf{h}(\mathbf{e}, \dot{\mathbf{e}})\| \leq k_{h_{1}}\|\dot{\mathbf{e}}\|+k_{h_{2}}\left\|\mathbf{f}_{t h}(\mathbf{e})\right\|$ where

$$
\mathbf{f}_{t h}(\mathbf{e})=\left[\begin{array}{lll}
\tanh \left(e_{x}\right) & \ldots & \tanh \left(e_{\beta_{2}}\right)
\end{array}\right]^{T} \in \mathbb{R}^{6}
$$

is the hyperbolic tangent function which has the following properties for $\forall \mathbf{e}$, $\dot{\mathbf{e}}$ :

$$
\begin{aligned}
& \left\|\mathbf{f}_{t h}(\mathbf{e})\right\| \leq\|\mathbf{e}\| \\
& \left\|\mathbf{f}_{t h}(\mathbf{e})\right\| \leq \sqrt{6} \\
& \left\|\mathbf{f}_{t h}(\mathbf{e})\right\|^{2} \leq \mathbf{f}_{t h}(\mathbf{e})^{T} \mathbf{e} \\
& \left\|\dot{\mathbf{f}}_{t h}(\mathbf{e})\right\| \leq\|\dot{\mathbf{e}}\|
\end{aligned}
$$

\section{APPENDIX B}

\section{LINEARIZATION FOR SYSTEM OBSERVABILITY}

As the dynamics of the system is highly non linear and coupled, a linearization of Eq.(12) is needed to prove local observability at a given operational point $\boldsymbol{\chi}_{\star}^{e}, \mathbf{U}_{\star}$. Notice that the observation equation (Eq.(28)) is already linear. In this sense, considering a Taylor series approximation and neglecting the high-order terms, the linearization of Eq.(12) is given in the form

$$
\Delta \dot{\boldsymbol{\chi}}^{e}=A_{l}^{e} \boldsymbol{\Delta} \boldsymbol{\chi}^{e}+B_{l}^{e} \boldsymbol{\Delta} \mathbf{U}
$$

such that $\Delta \dot{\boldsymbol{\chi}}^{e}=\dot{\boldsymbol{\chi}}^{e}-\mathbf{F}\left(\boldsymbol{\chi}_{\star}^{e}, \mathbf{U}_{\star}\right) \in \mathbb{R}^{18}, \boldsymbol{\Delta} \boldsymbol{\chi}^{e}=\boldsymbol{\chi}^{e}-\boldsymbol{\chi}_{\star}^{e} \in \mathbb{R}^{18}$, $\mathbf{\Delta} \mathbf{U}=\mathbf{U}-\mathbf{U}_{\star} \in \mathbb{R}^{6}$ and

$$
A_{l}^{e}=\left.\frac{\partial \mathbf{F}\left(\boldsymbol{\chi}^{e}, \mathbf{U}\right)}{\partial \boldsymbol{\chi}^{e}}\right|_{\boldsymbol{\chi}_{\star}^{e}, \mathbf{U}_{\star}} \quad ; \quad B_{l}^{e}=\left.\frac{\partial \mathbf{F}\left(\boldsymbol{\chi}^{e}, \mathbf{U}\right)}{\partial \mathbf{U}}\right|_{\boldsymbol{\chi}_{\star}^{e}, \mathbf{U}_{\star}}
$$


where $\boldsymbol{\chi}_{\star}^{e} \in \mathbb{R}^{18}$ and $\mathbf{U}_{\star} \in \mathbb{R}^{6}$ represent the operational point at which the linearization is made and $A_{l}^{e} \in \mathbb{R}^{18 \times 18}, B_{l}^{e} \in \mathbb{R}^{18 \times 6}$. Since the observability proof implies only the knowledge of $A_{l}^{e}$ and $C_{k}^{e}$, we exclusively present the expression that describes $A_{l}^{e}$ for its ulterior evaluation. Following the same procedure introduced in Section III to redefine $Z$ as in Eq.(26). From the dynamics of $\mathbf{q}$ given in Eq.(12) we found that:

$$
\begin{aligned}
& \frac{\partial \dot{\mathbf{q}}}{\partial \boldsymbol{\chi}^{e}}=\left[\begin{array}{lll}
\frac{\partial \dot{\mathbf{q}}}{\partial \mathbf{q}} & \frac{\partial \dot{\mathbf{q}}}{\partial \dot{\mathbf{q}}} & \frac{\partial \dot{\mathbf{q}}}{\partial \boldsymbol{\rho}}
\end{array}\right]=\left[\begin{array}{lll}
\mathbf{0} & \mathbf{I} & \mathbf{0}
\end{array}\right] \in \mathbb{R}^{6 \times 18} \\
& \frac{\partial \ddot{\mathbf{q}}}{\partial \boldsymbol{\chi}^{e}}=\left[\begin{array}{lll}
\frac{\partial \ddot{\mathbf{q}}}{\partial \mathbf{q}} & \frac{\partial \ddot{\mathbf{q}}}{\partial \dot{\mathbf{q}}} & \frac{\partial \ddot{\mathbf{q}}}{\partial \boldsymbol{\rho}}
\end{array}\right] \in \mathbb{R}^{6 \times 18} \\
& \frac{\partial \dot{\boldsymbol{\rho}}}{\partial \boldsymbol{\chi}^{e}}=\left[\begin{array}{lll}
\frac{\partial \dot{\boldsymbol{\rho}}}{\partial \mathbf{q}} & \frac{\partial \dot{\boldsymbol{\rho}}}{\partial \dot{\mathbf{q}}} & \frac{\partial \dot{\boldsymbol{\rho}}}{\partial \boldsymbol{\rho}}
\end{array}\right]=\left[\begin{array}{lll}
\mathbf{0} & \mathbf{0} & \mathbf{0}
\end{array}\right] \in \mathbb{R}^{6 \times 18}
\end{aligned}
$$

To extend the definition of the partial derivative in Eq. (64), let one recall $\ddot{\mathbf{q}}$ given in Eq. (19), such that the partial derivatives can be rewritten as:

$$
\frac{\partial \ddot{\mathbf{q}}}{\partial \dot{\mathbf{q}}}=-M(\mathbf{q})^{-1} C_{\dot{q}} \quad \frac{\partial \ddot{\mathbf{q}}}{\partial \mathbf{q}}=\ddot{Q}_{q} \quad \frac{\partial \ddot{\mathbf{q}}}{\partial \boldsymbol{\rho}}=M(\mathbf{q})^{-1}
$$

where

$$
\begin{aligned}
C_{\dot{q}} & =\left[\begin{array}{llll}
\frac{\partial C(\mathbf{q}, \dot{\mathbf{q}}) \dot{\mathbf{q}}}{\partial \dot{x}} & \frac{\partial C(\mathbf{q}, \dot{\mathbf{q}}) \dot{\mathbf{q}}}{\partial \dot{z}} & \ldots & \frac{\partial C(\mathbf{q}, \dot{\mathbf{q}}) \dot{\mathbf{q}}}{\partial \dot{\beta}_{2}}
\end{array}\right] \in \mathbb{R}^{6 \times 6} \\
\ddot{Q}_{q} & =\frac{1}{d_{M}}\left(A_{M_{q}}-\ddot{\mathbf{q}} \mathbf{d}_{M_{q}}\right)+M(\mathbf{q})^{-1} \mathbf{v}_{q} \in \mathbb{R}^{6 \times 6} \\
A_{M_{q}} & =\left[\begin{array}{lllll}
\frac{\partial A_{M}^{T}}{\partial x} \mathbf{v} & \frac{\partial A_{M}^{T}}{\partial z} \mathbf{v} & \ldots & \frac{\partial A_{M}^{T}}{\partial \beta_{1}} \mathbf{v} & \frac{\partial A_{M}^{T}}{\partial \beta_{2}} \mathbf{v}
\end{array}\right] \in \mathbb{R}^{6 \times 6} \\
\mathbf{d}_{M_{q}} & =\left[\begin{array}{lllll}
\frac{\partial d_{M}}{\partial x} & \frac{\partial d_{M}}{\partial z} & \ldots & \frac{\partial d_{M}}{\partial \beta_{1}} & \frac{\partial d_{M}}{\partial \beta_{2}}
\end{array}\right] \in \mathbb{R}^{1 \times 6} \\
\mathbf{v}_{q} & =\left[\begin{array}{lllll}
\frac{\partial \mathbf{v}}{\partial x} & \frac{\partial \mathbf{v}}{\partial z} & \ldots & \frac{\partial \mathbf{v}}{\partial \beta_{1}} & \frac{\partial \mathbf{v}}{\partial \beta_{2}}
\end{array}\right] \in \mathbb{R}^{6 \times 6}
\end{aligned}
$$

Such that $A_{l}^{e}$ evaluated at $\boldsymbol{\chi}_{\star}^{e}, \mathbf{U}_{\star}$ is defined as:

$$
A_{l}^{e}=\left.\left[\begin{array}{ccc}
\mathbf{0} & \mathbf{I} & \mathbf{0} \\
\ddot{Q}_{q} & -M(\mathbf{q})^{-1} C_{\dot{q}} & M(\mathbf{q})^{-1} \\
\mathbf{0} & \mathbf{0} & \mathbf{0}
\end{array}\right]\right|_{\boldsymbol{\chi}_{\star}^{e}, \mathbf{U}_{\star}}
$$

\section{REFERENCES}

[1] H. Shakhatreh, A. H. Sawalmeh, A. Al-Fuqaha, Z. Dou, E. Almaita, I. Khalil, N. S. Othman, A. Khreishah, and M. Guizani, "Unmanned aerial vehicles (uavs): A survey on civil applications and key research challenges," IEEE Access, vol. 7, pp. 48 572-48 634, 2019.

[2] Z. Hou, W. Wang, G. Zhang, and C. Han, "A survey on the formation control of multiple quadrotors," in Ubiquitous Robots and Ambient Intelligence (URAI), 2017 14th International Conference on. IEEE, 2017, pp. 219-225.

[3] T. Cabreira, L. Brisolara, and P. R Ferreira, "Survey on coverage path planning with unmanned aerial vehicles," Drones, vol. 3, no. 1, p. 4 2019.

[4] J. Escareño, J. Castillo, W. Abassi, G. Flores, and K. Camarillo, "Navigation strategy in-flight retrieving and transportation operations for a rotorcraft mav," in 4th Workshop on Research, Education and Development of Unmanned Aerial Systems (RED-UAS 2017), 2017, pp elec-proc.

[5] G. V. Raffo and M. M. d. Almeida, "A load transportation nonlinear control strategy using a tilt-rotor uav," Journal of Advanced Transportation, vol. 2018, 2018 .

[6] K. M. Cabral, S. R. B. dos Santos, S. N. Givigi, and C. L. Nascimento, "Design of model predictive control via learning automata for a single uav load transportation," in Systems Conference (SysCon), 2017 Annual IEEE International. IEEE, 2017, pp. 1-7.

[7] S. Barawkar, M. Radmanesh, M. Kumar, and K. Cohen, "Fuzzy logic based variable damping admittance control for multi-uav collaborative transportation," in 2018 Annual American Control Conference (ACC). IEEE, 2018, pp. 2084-2089.
[8] J. Alvarez-Muñoz, J. Castillo-Zamora, J. Escareno, I. Boussaada, F. Méndez-Barrios, and O. Labbani-Igbida, "Time-delay control of a multi-rotor vtol multi-agent system towards transport operations," in 2019 International Conference on Unmanned Aircraft Systems (ICUAS). IEEE, 2019, pp. 276-283.

[9] A. Fotouhi, H. Qiang, M. Ding, M. Hassan, L. G. Giordano, A. GarciaRodriguez, and J. Yuan, "Survey on uav cellular communications: Practical aspects, standardization advancements, regulation, and security challenges," IEEE Communications Surveys \& Tutorials, 2019.

[10] K. Low, T. Hu, S. Mohammed, J. Tangorra, and M. Kovac, "Perspectives on biologically inspired hybrid and multi-modal locomotion," Bioinspiration \& biomimetics, vol. 10, no. 2, p. 020301, 2015.

[11] J. Tang, B. Li, Z. Li, and J. Chang, "A novel underwater snake-like robot with gliding gait," in 2017 IEEE 7th Annual International Conference on CYBER Technology in Automation, Control, and Intelligent Systems (CYBER). IEEE, 2017, pp. 1113-1118.

[12] Y. S. Hagh, R. M. Asl, and V. Cocquempot, "A hybrid robust fault tolerant control based on adaptive joint unscented kalman filter," ISA transactions, vol. 66, pp. 262-274, 2017.

[13] S. Refoufi and K. Benmahammed, "Control of a manipulator robot by neuro-fuzzy subsets form approach control optimized by the genetic algorithms," ISA transactions, vol. 77, pp. 133-145, 2018.

[14] P. Londhe, Y. Singh, M. Santhakumar, B. Patre, and L. Waghmare, "Robust nonlinear pid-like fuzzy logic control of a planar parallel (2prpppr) manipulator," ISA transactions, vol. 63, pp. 218-232, 2016.

[15] L. Angel and J. Viola, "Fractional order pid for tracking control of a parallel robotic manipulator type delta," ISA transactions, vol. 79, pp. $172-188,2018$

[16] D. Six, S. Briot, A. Chriette, and P. Martinet, "The kinematics, dynamics and control of a flying parallel robot with three quadrotors," IEEE Robotics and Automation Letters, vol. 3, no. 1, pp. 559-566, 2018.

[17] M. Zhao, T. Anzai, F. Shi, X. Chen, K. Okada, and M. Inaba, "Design, modeling, and control of an aerial robot dragon: A dual-rotorembedded multilink robot with the ability of multi-degree-of-freedom aerial transformation," IEEE Robotics and Automation Letters, vol. 3, no. 2, pp. 1176-1183, 2018.

[18] M. Zhao, K. Kawasaki, K. Okada, and M. Inaba, "Transformable multirotor with two-dimensional multilinks: modeling, control, and motion planning for aerial transformation," Advanced Robotics, vol. 30, no. 13 , pp. 825-845, 2016.

[19] M. Zhao, F. Shi, T. Anzai, K. Chaudhary, X. Chen, K. Okada, and M. Inaba, "Flight motion of passing through small opening by dragon: Transformable multilinked aerial robot," in 2018 IEEE/RSJ International Conference on Intelligent Robots and Systems (IROS). IEEE, 2018, pp. 4735-4742.

[20] T. Anzai, M. Zhao, X. Chen, F. Shi, K. Kawasaki, K. Okada, and M. Inaba, "Multilinked multirotor with internal communication system for multiple objects transportation based on form optimization method," in Intelligent Robots and Systems (IROS), 2017 IEEE/RSJ International Conference on. IEEE, 2017, pp. 5977-5984.

[21] J. Castillo-Zamora, J. Escareno, I. Boussaada, O. Labbani, and K. Camarillo, "Modeling and control of an aerial multi-cargo system: Robust acquiring and transport operations," in 2019 18th European Control Conference (ECC). IEEE, 2019, pp. 1708-1713.

[22] J. J. Castillo-Zamora, J. Escareno, J. Alvarez, J. Stéphant, and I. Boussaada, "Disturbances and coupling compensation for trajectory tracking of a multi-link aerial robot," in 2019 6th International Conference on Control, Decision and Information Technologies (CoDIT), April 2019, pp. $738-743$.

[23] S. Park, Y. Lee, J. Heo, and D. Lee, "Pose and posture estimation of aerial skeleton systems for outdoor flying," in 2019 International Conference on Robotics and Automation (ICRA). IEEE, 2019, pp. 704-710.

[24] E. Gauterin, P. Kammerer, M. Kühn, and H. Schulte, "Effective wind speed estimation: Comparison between kalman filter and takagi-sugeno observer techniques," ISA transactions, vol. 62, pp. 60-72, 2016.

[25] F. Shi, M. Zhao, T. Anzai, X. Chen, K. Okada, and M. Inaba, "External wrench estimation for multilink aerial robot by center of mass estimator based on distributed imu system," in 2019 International Conference on Robotics and Automation (ICRA). IEEE, 2019, pp. 1891-1897.

[26] O. Mofid and S. Mobayen, "Adaptive sliding mode control for finitetime stability of quad-rotor uavs with parametric uncertainties," ISA transactions, vol. 72, pp. 1-14, 2018.

[27] F. A. Goodarzi and T. Lee, "Global formulation of an extended kalman filter on se (3) for geometric control of a quadrotor uav," Journal of Intelligent \& Robotic Systems, vol. 88, no. 2-4, pp. 395-413, 2017. 
[28] Y. Zheng, L. Dong, and Q. Wang, "Multi-rotor uav attitude calculation based on extended kalman filter," in 2018 Chinese Control And Decision Conference (CCDC). IEEE, 2018, pp. 478-483.

[29] M. Ghanai, A. Medjghou, and K. Chafaa, "Extended kalman filter based states estimation of unmanned quadrotors for altitude-attitude tracking control," 2018.

[30] A. Nez, L. Fradet, F. Marin, T. Monnet, and P. Lacouture, "Identification of noise covariance matrices to improve orientation estimation by kalman filter," Sensors, vol. 18, no. 10, p. 3490, 2018.

[31] G. Reina and A. Messina, "Vehicle dynamics estimation via augmented extended kalman filtering," Measurement, vol. 133, pp. 383-395, 2019

[32] A. Paul, I. Kamwa, and G. Joos, "Centralized dynamic state estimation using a federation of extended kalman filters with intermittent pmu data from generator terminals," IEEE Transactions on Power Systems, vol. 33 , no. 6, pp. 6109-6119, 2018.

[33] N. Ko, W. Youn, I. Choi, G. Song, and T. Kim, "Features of invariant extended kalman filter applied to unmanned aerial vehicle navigation," Sensors, vol. 18, no. 9, p. 2855, 2018.

[34] S. Driessen, N. Janssen, L. Wang, J. Palmer, and H. Nijmeijer, "Experimentally validated extended kalman filter for uav state estimation using low-cost sensors," IFAC-PapersOnLine, vol. 51, no. 15, pp. 43-48, 2018

[35] H. A. Pointon, B. J. McLoughlin, C. Matthews, and F. A. Bezombes, "Towards a model based sensor measurement variance input for extended kalman filter state estimation," Drones, vol. 3, no. 1, p. 19, 2019.

[36] V. Lertpiriyasuwat, M. C. Berg, and K. W. Buffinton, "Extended kalman filtering applied to a two-axis robotic arm with flexible links," The International Journal of Robotics Research, vol. 19, no. 3, pp. 254-270, 2000.

[37] R. Kelly, V. S. Davila, and J. A. L. Perez, Control of robot manipulators in joint space. Springer Science \& Business Media, 2006.

[38] J. J. Castillo-Zamora, K. A. Camarillo-Gómez, G. I. Pérez-Soto, and J. Rodríguez-Reséndiz, "Comparison of pd, pid and sliding-mode position controllers for v-tail quadcopter stability," IEEE Access, vol. 6, pp. 38 086-38 096, 2018.

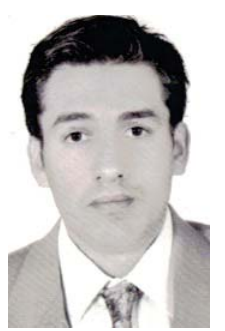

José J. Castillo-Zamora holds a doctoral researcher position at IPSA Paris, Ivry-sur-Seine, France. He is also a Ph. D. student at L2S of Université Paris Sud-CNRS-CentraleSupelec, Université Paris Saclay, Gif-sur-Yvette, France. He received his Master degree in Mechanical Engineering in 2016 at Tecnológico Nacional de México en Celaya. He worked as a signature professor at the departments of mechanical and mechatronics engineering, both, of the Tecnológico Nacional de México en Celaya in 2015 and as an associate professor at Mechatronics Engineering Department from 2016 to 2018. His current research fields are the modeling, control and design of intelligent aerial and underwater systems; and Time-delay Control applications.

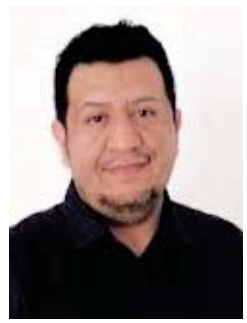

Juan Escareno received a Ph.D. (2008) in Automatic Control from the HEUDIASYC laboratory of the University of Technology of Compiegne (UTC), France. From 2008 to 2010, he held a Post-Doctoral fellowship at the "Unité Mixte de Internationale du CNRS - UMI 3175". He was a CNRS project researcher at the UTC, from 2010 to 2012. In 2012, he has held a four-month stay as a visiting scientist at the French Nuclear Energy Commission (CEA). He served, from 2012 to 2013, as a PostDoctoral Research Associate at the department of "Automatique et Systèmes Micro-Mécatroniques" (AS2M) at FEMTO-ST UMR CNRS 6174, France. From 2014-2018, he held an associate professor position at the "Institut Polytechnique des Sciences Avancées" at Ivry-surSeine, France. Since 2018, he is associate professor at XLim CNRS Research Institute and ENSIL-ENSCI Engineering School at University of Limoges. His current research encompasses nonlinear control of multi-agent systems, hybdrid and interactive robotic systems and autonomous robust navigation.

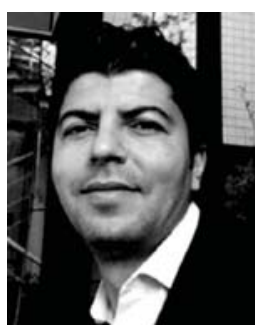

Islam Boussaada received the M.Sc. degree in mathematics from University Tunis II, Tunis, Tunisia, as well as an M.Sc. degree in pure mathematics from University Paris 7, Paris, France, in 2004, the Ph.D. degree in mathematics from Normandy University, Normandy, France, in December 2008, and the French Habilitation (HDR) in physics from the University of Paris-Saclay, Saint-Aubin, France, in 2016. In 2010, he served for two years as a Postdoctoral Fellow in control of time-delay systems with the Laboratory of Signals and Systems (L2S), CentraleSupélec-CNRS-Université Paris Sud, Gif-sur-Yvette, France. Since 2012, he has been an Associate Professor with IPSA and an Associate Researcher with L2S, University of Paris-Saclay, CentraleSupélec-CNRSUniversity Paris Sud. Since 2016, he has been an Associate Member with Inria Saclay DISCO project. His current research focuses on the qualitative theory of dynamical systems and its application in control. It covers the analysis of the delay effect on dynamics, stability, and stabilization of delay systems and hyperbolic partial differential equations, oscillations, and periodic solutions of functional differential equations, control of vibrations.

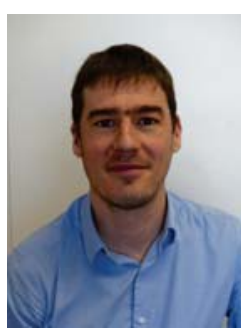

Joanny Stéphant was born in Lorient, France, in 1978. He received the master title from the Technology University of Compiegne (UTC), Compiègne, France in 2001 with his engineer diploma. In 2004 he obtained a Ph.D. degree in automatic control and application to vehicle dynamics with the Heudiasyc Lab. Since 2005 he is an assistant professor in the Mechatronics department in the national engineering school in Limoges (ENSIL-ENSCI). His research areas deals with vehicle dynamics, mobile robotics, automatic control, observers, observability.

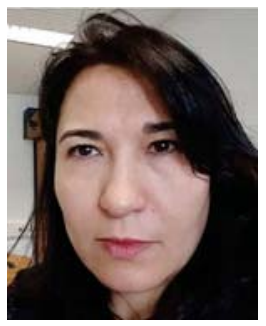

Ouiddad Lagbanni received the Eng. and $\mathrm{PhD}$ Degrees in robotics from the ENSMM-University of Franche-Comte, and the Habilitation Degree in vision and robotics from the University of Picardie Jules Verne, France. Since 2013, she has been Full Professor with XLim CNRS Institute of Research and ENSIL-ENSCI Engineering School at University of Limoges. She is the Head of the Mechatronics Department at ENSIL-ENSCI Engineering School and supervises the Robotics and Mechatronics research group at the XLim institute. Her main research interests are in the field of robotics, including enactive perception and control, omnidirectional catadioptric vision, cooperative robotics, and localization and navigation of autonomous systems. 